OPEN ACCESS

Edited by:

Bryan D. Devan,

Towson University, United States

Reviewed by:

Niels Niethard,

University of Tübingen, Germany

Adonis Yiannakas,

University of Bern, Switzerland

*Correspondence:

Sara J. Aton

saton@umich.edu

Received: 30 July 2021

Accepted: 26 August 2021

Published: 13 September 2021

Citation:

Raven F and Aton SJ (2021) The

Engram's Dark Horse:

How Interneurons Regulate State-Dependent Memory Processing

and Plasticity.

Front. Neural Circuits 15:750541. doi: 10.3389/fncir.2021.750541

\section{The Engram's Dark Horse: How Interneurons Regulate State-Dependent Memory Processing and Plasticity}

\author{
Frank Raven and Sara J. Aton* \\ Department of Molecular, Cellular, and Developmental Biology, College of Literature, Sciences, and the Arts, University
} of Michigan, Ann Arbor, MI, United States

Brain states such as arousal and sleep play critical roles in memory encoding, storage, and recall. Recent studies have highlighted the role of engram neuronspopulations of neurons activated during learning-in subsequent memory consolidation and recall. These engram populations are generally assumed to be glutamatergic, and the vast majority of data regarding the function of engram neurons have focused on glutamatergic pyramidal or granule cell populations in either the hippocampus, amygdala, or neocortex. Recent data suggest that sleep and wake states differentially regulate the activity and temporal dynamics of engram neurons. Two potential mechanisms for this regulation are either via direct regulation of glutamatergic engram neuron excitability and firing, or via state-dependent effects on interneuron populationswhich in turn modulate the activity of glutamatergic engram neurons. Here, we will discuss recent findings related to the roles of interneurons in state-regulated memory processes and synaptic plasticity, and the potential therapeutic implications of understanding these mechanisms.

Keywords: hippocampus, neocortical circuits, oscillations, sleep, interneurons, replay, neuronal reactivation

\section{INTRODUCTION-ENGRAM NEURONS IN CONTEXT}

Animals have the fundamental ability to encode, store, and retrieve information about the world around them, which is crucial for their survival. Initial memory formation is thought to rely on the activation of neurons across multiple brain regions. This set of neurons-activated during experience and reactivated upon recall-is commonly referred to as the memory trace or engram.

\footnotetext{
Abbreviations: $\mathrm{AD}$, Alzheimer's disease; $\mathrm{A} \beta$, amyloid- $\beta$; $\mathrm{BDNF}$, brain-derived neurotrophic factor; $\mathrm{CA}$, cornu ammonis; CCK, cholecystokinin; CFC, contextual fear conditioning; CFM, contextual fear memory; ChR2, channelrhodopsin; CSEA, cell type-specific enrichment analysis; DG, dentate gyrus; FS, fast-spiking; GABA, gamma aminobutyric acid; IEG, immediate-early gene; LTP, long-term potentiation; LTD, long-term depression; M1, primary motor cortex; MDD, major depressive disorder; mPFC, medial prefrontal cortex; mRNA, messenger ribonucleic acid; NMDA, $N$-methyl-D-aspartate; nNOS, neuronal nitric oxide synthase; NPY, neuropeptide Y; NREM, non-rapid eye movement; PC, principal (glutamatergic) celli; PV, parvalbumin; REM, rapid eye movement; SD, sleep deprivation; SO, stratum oriens; SP, stratum pyramidale; SR, stratum radiatum; SST, somatostatin; TRAPs, targeted recombination in activated populations; V1, primary visual cortex; VIP, vasoactive intestinal peptide; WGCNA, weighted gene co-expression network analysis.
} 
Though the idea of the engram was postulated about a century ago (Semon, 1921), it is only over the past decade that engram neuron populations have been identified by researchers and their function manipulated, due to recent technological advances. The first studies of this kind focused on neuron populations encoding contextual information in the hippocampus, a brain structure in the medial temporal lobe which is crucial for long-term spatial and episodic memory storage. Input to the hippocampus from multiple neocortical structures, including sensory cortices, flows via the entorhinal cortex perforant pathway into the dentate gyrus (DG). Information from DG (typically encoded by a sparse granule cell neuron population) is relayed via mossy fibers to hippocampal subarea cornu ammonis 3 (CA3); CA3 pyramidal neurons project via the Schaffer collaterals to hippocampal area CA1; CA1 pyramidal neurons project to the hippocampal subiculum (and to amygdala and entorhinal cortex); subicular pyramidal neurons provide the major output to the entorhinal cortex and other neocortical structures (as well as subcortical structures such as the amygdala). This relatively simple feedforward excitatory circuit is capable of encoding and at least transiently storing a vast amount of information related to space, sensation, and sequence of events. Critically, however, each substructure has recurrent connections, and reciprocal communication between hippocampus and neocortex-which may lead to information elaboration, interaction, or modification over time (Nadel et al., 2007; Dudai, 2012; Moscovitch et al., 2016; Barron et al., 2017; Hardt and Sossin, 2020). Principal neurons (DG granule cells and CA1/CA3/subiculum pyramidal cells) in the hippocampus, as well as the neocortex, are known to be activated during new experiences, such as those leading to de novo associative learning. This phenomenon is easily observed using immediate-early gene (IEG) expression (e.g., Arc, Cfos, or Npas4), as a readout measure. Principal neurons in both neocortex and hippocampus also undergo ultrastructural and intracellular molecular changes in the hours to days following learning (O’Malley et al., 1998; Trabalza et al., 2012; Yang and Gan, 2012; Alberini and Kandel, 2014; Lu and Zou, 2017; Sliwinski et al., 2020). These changes have downstream effects on the strength of synaptic connections between neurons (Whitlock et al., 2006; Cooke and Bear, 2010, 2014), neuronal activity (Thompson et al., 1996; Ognjanovski et al., 2014, 2017; Durkin and Aton, 2016; Clawson et al., 2021), and biosynthetic/metabolic changes (Im et al., 2009; Koberstein et al., 2018; Rao-Ruiz et al., 2019).

Recently developed genetic tools have allowed experimental access to the engram neuron populations that are selectively activated during specific learning events. Multiple intersectional genetic strategies have been developed to induce recombination in activated neurons, all of which are based on transgene expression from an IEG (Arc or Cfos) promoter (Reijmers et al., 2007; Liu et al., 2012; Guenthner et al., 2013). The first studies investigating the function of engram neurons used these tools to either identify or chemogenetically or optogenetically manipulate DG engram neurons encoding specific environmental contexts. The authors of these studies expressed channelrhodopsin (ChR2) in engram cells active during contextual fear conditioning (CFC) (placement in a novel context + delivery of a foot shock). They were then able to elicit "recall" of contextual fear memory (CFM), in which mice exhibited freezing responses to hippocampal light delivery, at a later time point-even when they were in a completely dissimilar context (Liu et al., 2012). In a related study, the authors found that by pairing a shock in one context with simultaneous optogenetic activation of engram neurons tagged to express ChR2 in a second, dissimilar context, they were able to generate a false associative memory of foot shock with the context used to induce recombination (Ramirez et al., 2013). These findings suggest that experimental activation of engram neuron populations is sufficient to evoke recall of specific memories (be they true or false). More recently, engram populations have been identified and manipulated in the neocortex as well (Marshel et al., 2019; Clawson et al., 2021); activation of these neocortical engram cells has a similar effect, of re-evoking experiences occurring during their genetic tagging.

Because the majority of engram neurons identified in these studies appear to be excitatory (e.g., having the morphology of neocortical pyramidal cells, DG granule cells), one would be forgiven for concluding that memory encoding, consolidation, and recall are the domain of glutamatergic circuits. However, GABAergic interneurons-the main source of inhibition in the hippocampus and neocortex-also play a critical role in mnemonic processing. Indeed, one of the IEGs described above, Npas4, which is transcribed in response to learning experiences, is known to play different roles in GABAergic and glutamatergic neurons, resulting in alterations in both excitatory-to-inhibitory and inhibitory-to-excitatory neuronal connectivity (Spiegel et al., 2014). A more recent study suggests that distinct engram populations exist (for example, among DG granule cells activated during CFC), which show heightened expression of either the IEG Cfos or Npas4 after CFC-but not both (Sun et al., 2020). Intriguingly, these populations differ in regard to their inhibitory input-with Npas4-expressing engram neurons having comparatively higher inhibitory drive. An even more interesting feature of these DG populations is that they may play different roles in CFM recall. The authors of this study found that the Npas-expressing engram population (which received greater inhibition) was more active when mice were discriminating between the CFM context and a similar context during recall. They also found that chemogenetic suppression of activity in the Npas-expressing population (but not the Cfos-expressing population), disrupted context discrimination. Conversely, inhibition of the Cfos-expressing engram population (which received less inhibition) increased contextual fear discrimination, and activation of this population increased contextual fear generalization (Sun et al., 2020). These findings beg the question of how changes in connections between excitatory and inhibitory neurons affect the nature of, and activity among, neurons representing specific memories.

GABAergic interneurons represent about $15-20 \%$ of total neurons in hippocampus and neocortex, and are highly heterogeneous. Various interneuron types have been classified based on anatomical location, structural morphology and biochemical properties (Pelkey et al., 2017; Booker and Vida, 2018; Lourenco et al., 2020). Within both the hippocampus and neocortex, broad subclasses have been identified based on 
biomarker expression: those that express parvalbumin (PV+), those that express somatostatin (SST+), those that express vasoactive intestinal peptide (VIP+), and those that express cholecystokinin (CCK+). Note each of these have different subtypes, some of which are further delineated based on gene expression patterns. For example, some SST+ interneurons coexpress neuropeptide Y (NPY), and some NPY+ interneurons co-express neuronal nitric oxide synthase (nNOS). Some VIP+ interneurons are also CCK+. While the functions of many of these specific subclasses are still understudied, there is significant recent data to suggest that $\mathrm{PV}+$ and SST+ interneurons make major contributions to memory encoding and storage. Here we will discuss how interneurons contribute to the process of memory encoding and storage in the brain, to shape the engram. Because there is a growing appreciation of the roles of sleep states in promoting memory storage, and their roles more broadly in regulating inhibitory transmission in the brain (Puentes-Mestril and Aton, 2017; Puentes-Mestril et al., 2019), we will focus our discussion on sleep-dependent consolidation mechanisms. We will describe recent evidence which indicates differential roles for interneuron function in brain states-wake, non-rapid eye movement (NREM) sleep, and rapid eye movement (REM) sleep-in regulation of memory processing.

\section{PV+ AND SST+ INTERNEURONS ARE CRITICAL REGULATORS OF MEMORY ENCODING, STORAGE, AND RECALL}

As mentioned above, a large proportion of GABAergic interneurons in both the neocortex and hippocampus are either PV+ or SST+. These two interneuron populations both provide strong inhibition to neighboring excitatory (e.g., pyramidal or granule) neurons' cell bodies/axon initial segments and dendrites, respectively (Booker and Vida, 2018; Lourenco et al., 2020). As discussed in more detail below, both populations receive inhibitory input from VIP+ interneurons (a subclass of selectively interneuron-targeting GABAergic neurons). This basic microcircuit motif recurs throughout the hippocampus and neocortical layers (Figure 1).

Parvalbumin interneurons constitute the largest interneuron subpopulation in the forebrain, representing roughly 40 and $25 \%$ of all GABAergic interneurons in the neocortex and hippocampal CA1, respectively (Rudy et al., 2011; Bezaire and Soltesz, 2013). Each of these interneurons typically innervate a large number of neighboring principal neurons' perisomatic regions, exerting strong control over their firing, and contribute to both feedforward and feedback inhibition (Udakis et al., 2020). Within the hippocampus, typically fast-spiking (FS) $\mathrm{PV}+$ interneurons are implicated in a number of memory processes. $\mathrm{PV}+$ interneurons in $\mathrm{CA} 1$ and $\mathrm{CA} 3$ are activated following CFC (Donato et al., 2013; Ognjanovski et al., 2014, 2017; Xia et al., 2017). Disruption of CA1 PV+ interneurons' output via targeted expression of tetanus toxin light chain leads to deficits in hippocampus-dependent spatial working memory, but leaves cortically mediated reference memory unaffected (Murray et al., 2011). As shown more recently by our lab and others, post-CFC chemogenetic or optogenetic inhibition of PV+ interneurons [in either dorsal hippocampus or medial prefrontal cortex (mPFC)] impairs CFM consolidation (Ognjanovski et al., 2017, 2018; Xia et al., 2017). A number of studies have characterized intracellular signaling pathways that must be activated within PV+ interneurons in order to support memory storage. These pathways include those known to be required for Hebbian synaptic plasticity mechanisms [i.e., long-term potentiation (LTP) and LTD]. Cell type-specific ablation of $N$-methyl-D-aspartate (NMDA) receptors in PV+ interneurons disturbs working, short-term, and long-term spatial memory (Korotkova et al., 2010). PV+ interneuron targeted knockout of $\gamma$ CaMKII leads to impaired consolidation of both hippocampus-dependent CFM and inhibitory avoidance, but critically, preserves consolidation of hippocampus-independent tone-cued fear memory (He et al., 2021). PV+ interneurontargeted knockdown of brain-derived neurotrophic factor (BDNF) receptor TrkB disrupts short-term spatial working memory-although this phenotype is seen only in male mice (Grech et al., 2019). CFM consolidation relies on activity of D1/5 dopamine receptors, and downstream activation of cAMP and ERK, in hippocampal PV+ interneurons in the hours following CFC (Karunakaran et al., 2016). PV+ interneurons in various brain structures, including the insular cortex, medial septum, and nucleus accumbens also play critical roles during memory recallcoordinating activity within limbic structures during recall of appetitive and aversive associations (Trouche et al., 2019; SansDublanc et al., 2020; Yiannakas et al., 2021). Altogether, these data highlight the importance of PV+ interneurons for memory processing, and underscore that the connectivity between PV+ interneurons and the principal cells they target is crucial for engram formation.

Somatostatin interneurons comprise the second-largest population of forebrain GABAergic neurons (Rudy et al., 2011; Bezaire and Soltesz, 2013). Somatostatin (a.k.a., growth hormone-inhibiting hormone or somatotropin release inhibitory factor) is released along with GABA in this diverse population of interneurons (Pelkey et al., 2017; Booker and Vida, 2018; Honore et al., 2021) and activates Gi/o-coupled receptors (Gunther et al., 2018). Axons of SST+ interneurons target the dendritic compartments neighboring principal neurons, providing a source of strong inhibition. Like PV+ interneurons, SST+ interneurons have been implicated in regulating hippocampusdependent memory processes. However, based on the available data, it seems likely that the two populations play distinct roles in memory encoding and consolidation. For example, chemogenetic inhibition of SST+ interneurons in the DG during CFC improves CFM recall $24 \mathrm{~h}$ or even a full week later (Stefanelli et al., 2016). Chemogenetic activation of SST+ interneurons during CFC, in contrast, has no effect on CFM recall at $24 \mathrm{~h}$ post-learning, but disrupts remote CFM recall 1 week later. This bidirectional modulation of memory retention is mirrored by DG granule cell activation during remote recall; mice that are SST+ interneuron-inhibited or -activated during encoding have larger and smaller DG granule cell populations, respectively, activated during recall. Intriguingly, the same manipulations of DG PV+ interneurons' activity are without 

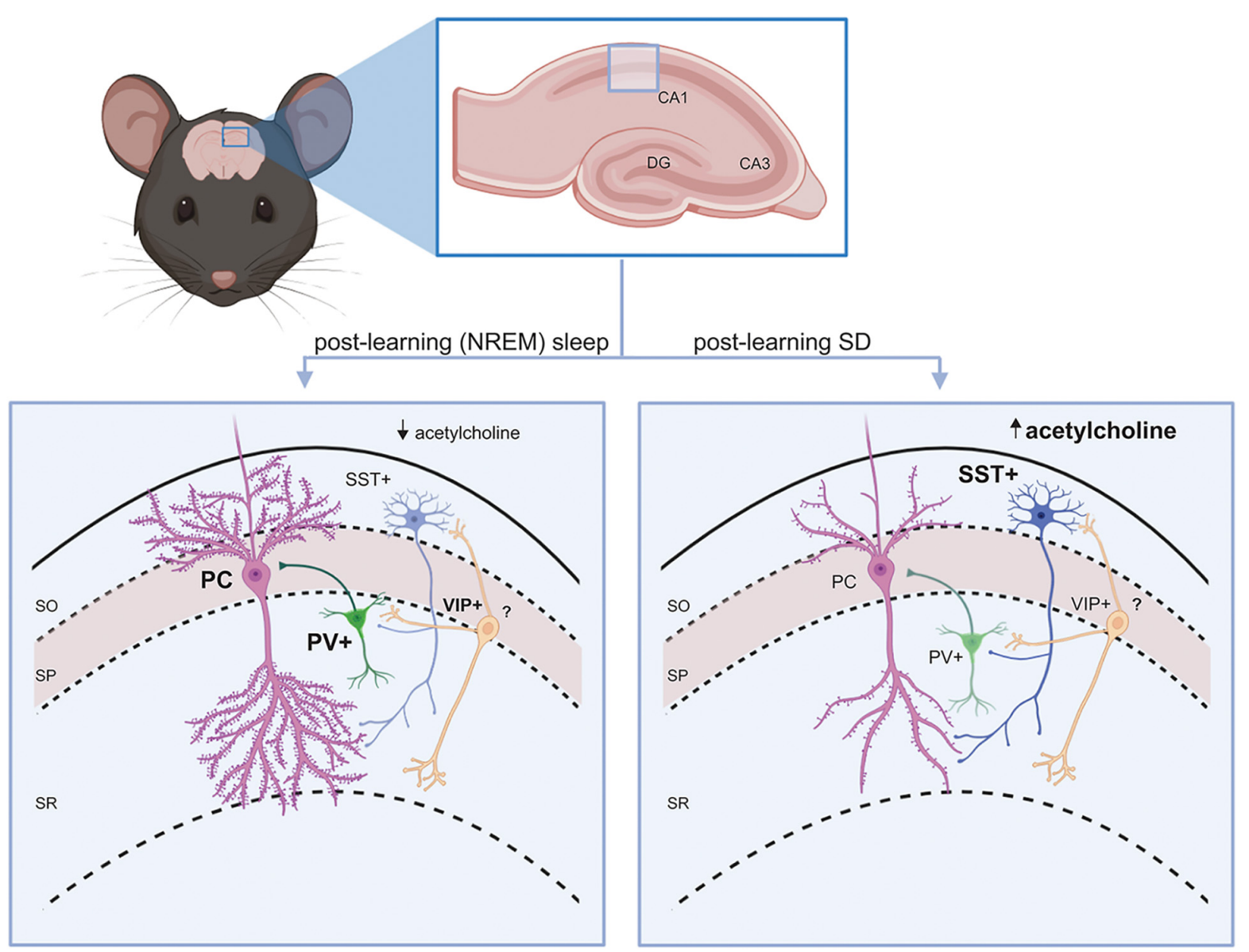

FIGURE 1 | Interneuron circuit motifs and their regulation by state. A conserved interneuron motif (present throughout hippocampus and neocortex) is shown in the mouse CA1. PV+ and SST+ interneurons provide inhibitory GABAergic input to principal (glutamatergic) cells' (PCs') soma/axon initial segment and dendrites, respectively. VIP+ interneurons inhibit SST+ interneurons in the circuit. Left: During a period of post-learning sleep, principal cells and PV+ interneurons generally become more active (Ognjanovski et al., 2014), while SST+ interneurons are relatively quiescent (due to lower acetylcholine-mediated activation in NREM sleep) (Delorme et al., 2021). Some principal neurons (engram neurons) will become selectively more active, and may form new and/or stronger synaptic connections (Clawson et al., 2021). PV+ interneurons promote more coherent NREM and REM sleep oscillations (Ognjanovski et al., 2017, 2018). All these features are essential components of sleep-dependent memory consolidation. Right: When learning is followed by SD, SST+ interneurons shown increased activity in response to higher acetylcholine levels (Delorme et al., 2021). VIP+ interneurons may also have reduced activation, reducing inhibitory input to SST+ interneurons. This leads to suppression of firing in surrounding principal neurons (Delorme et al., 2021). As a result, dendritic spines on engram neurons (and other principal neurons) will be reduced and connections between them will be weakened (Havekes et al., 2016; Raven et al., 2019; Havekes and Aton, 2020), which impairs memory consolidation.

effect on either behavioral recall or the DG network activation at recall (Stefanelli et al., 2016). In this same study, the authors found that DG SST+ interneurons, but not PV+ interneurons, are highly activated during initial memory encoding (e.g., exploring a novel context). This latter finding has recently been replicated in the prefrontal cortex, where glutamatergic synapses onto SST+ interneurons are actually potentiated by auditory-cued fear conditioning (Cummings and Clem, 2020). Based on SST+ interneurons' activation by activity in surrounding granule cells, and the effects of acute manipulations of their activity on granule cells, the authors concluded that these neurons support lateral inhibition between granule cells in the context of hippocampal memory encoding.

Consistent with the conclusions of the study described above, several pieces of recent data suggest that hippocampal SST+ interneurons may play a critical role in precisely encoding memories. For example, a recent study showed that optogenetic inhibition of DG SST+ interneurons during training on either a contextual or object-location discrimination task led to deficits at testing $24 \mathrm{~h}$ later, but only if the two scenarios being discriminated were highly similar (Morales et al., 2021). Another recent study tested the role of DG SST+ interneurons in the context of encoding foreground contextual fear, where context is the most salient predictor of a foot shock, vs. background contextual fear, where foot shocks are immediately preceded by a tone cue, making context itself less salient (Raza et al., 2017). Generally, freezing responses to the conditioning context itself are reduced when mice are background conditioned, relative to foreground conditioned mice. The authors found that training-targeted chemogenetic inactivation of DG SST+ 
interneurons increased the activation of surrounding DG granule cells during background conditioning, and selectively increased freezing responses to context reexposure $24 \mathrm{~h}$ after background conditioning (Raza et al., 2017). The studies described above suggest that SST+ interneuron activation during memory encoding effectively gates the activation of surrounding excitatory neurons. This constraining of the engram population size can limit the strength of encoding for simple associations, or in some instances, can improve memory precision and prevent generalization.

The critical role for SST+ interneurons during encoding begs the question of how these interneurons contribute to subsequent consolidation. Available data suggests that cellular pathways involved in Hebbian plasticity are engaged in SST+ interneurons by learning. For example, SST+ interneuron targeted disruption of either eIF2 or mTOR (key regulators of neuronal activityregulated translation required for structural plasticity) leads to impaired consolidation of multiple hippocampus-dependent forms of memory (Artinian et al., 2019; Sharma et al., 2020). A recent study from our lab (described in greater detail below) assessed the effects of chemogenetically activating or inhibiting DG SST+ interneurons over the hours following CFC (Delorme et al., 2021). We found that these manipulations were sufficient to disrupt or enhance CFM consolidation, as evidenced by decreased or increased contextual freezing responses, respectively, $24 \mathrm{~h}$ after training. Consistent with other recent findings on the function of SST + interneurons (described above), we found that chemogenetic activation or inhibition during the consolidation phase reduced or increased $\mathrm{cFos}$ expression among DG granule cells, respectively. These data suggest that inhibitory gating of activity in the DG network (and the engram neuron population) by SST + interneurons in the hours following learning constrains hippocampal memory consolidation.

This interpretation of our data is consistent with recent neuroanatomical findings from mouse neocortex in the context of consolidation of a recently learned motor task (Chen et al., 2015). The authors found that immediately following training on the task (i.e., during consolidation), axon terminals from SST+ interneurons onto distal dendrites of surrounding pyramidal neurons are gradually reduced. This same behavior was not seen for perisomatic inhibitory synapses from PV+ interneurons, which were generally increased, rather than decreased, as a function of motor training. Thus, inhibitory gating by SST+ interneurons may serve as a constraint to consolidation even outside of the hippocampus.

\section{STATE-DEPENDENT REGULATION OF INTERNEURON ACTIVITY DURING MEMORY CONSOLIDATION}

Sleep is vital to hippocampal and neocortical memory processing (Diekelmann and Born, 2010; Rasch and Born, 2013; PuentesMestril and Aton, 2017; Puentes-Mestril et al., 2019), with experimental sleep deprivation (SD) capable of disrupting encoding, consolidation, and recall (Yoo et al., 2007; Heckman et al., 2020). In mice, as little as a few hours of SD following learning profoundly disrupts consolidation for hippocampalmediated spatial and contextual tasks, e.g., object-location memory and CFM (Graves et al., 2003; Vecsey et al., 2009; Prince et al., 2014; Havekes et al., 2016; Ognjanovski et al., 2018). This disruption is associated with reductions in neurons dendritic spine density (Havekes et al., 2016; Raven et al., 2018; Havekes and Aton, 2020), protein synthesis (Seibt et al., 2012; Tudor et al., 2016), and intracellular signaling in pathways required for synaptic potentiation (Aton et al., 2009b; Vecsey et al., 2009; Bridi et al., 2015; Dumoulin et al., 2015). Our lab has recently demonstrated that $\mathrm{SD}$ also profoundly disrupts principal neuron activity in the hippocampus. We found that SD decreases neuronal activity-driven phosphorylation of ribosomal protein S6 throughout the dorsal hippocampus, suggesting decreased neuronal activity (Delorme et al., 2021). This is consistent with previous findings from our lab, where IEG Arc's messenger ribonucleic acid (mRNA) and protein expression is reduced in DG after a period of SD (Delorme et al., 2019). Together these findings are consistent with human brain imaging studies, which have shown that learning-associated activation of the hippocampus is disrupted by prior overnight SD (Yoo et al., 2007). In our study, we found that while CFC itself causes subsequent increases in S6 phosphorylation throughout the hippocampus (consistent with electrophysiological findings from our lab, see below) (Ognjanovski et al., 2014), SD in the hours following CFC reduces phosphorylation of S6 (Delorme et al., 2021). Taken together, these data suggest that hippocampal neurons' activity is dramatically suppressed by SD.

To clarify which hippocampal neurons remain active in the face of SD, we next used an unbiased bioinformaticsguided approach to profile mRNAs differentially associated with ribosomes containing phosphorylated S6 (i.e., those from the most active neurons) after sleep or SD. mRNAs which were associated with phosphorylated S6-containing ribosomes were sequenced from hippocampi of mice allowed ad lib sleep, or subjected to 3-h SD. Using weighted gene co-expression network analysis (WGCNA), we identified known clusters of co-regulated transcripts with abundance that varied in the hippocampus as a function of prior sleep and wake amounts. We found that two clusters of known co-regulated mRNAs are upregulated together on phosphorylated ribosomes, in proportion to prior wake time. We next compared these wakedriven, clustered transcripts with previously described cell typespecific transcript profiles, using cell type-specific enrichment analysis (CSEA). We found that mRNA markers of SST + and $\mathrm{NPY}+$ interneurons (but not VIP+ or PV+ interneurons) are selectively increased after SD (Figure 1), as are transcripts associated with cholinergic and orexinergic neurons. Thus, while $\mathrm{SD}$ appears to reduce activity of principal neurons (as mentioned above), it increases S6 phosphorylation in SST+ interneurons. These findings are consistent with a mechanism whereby during SD, strong inhibition from SST+ interneurons suppress activity in neighboring principal neurons. We then tested the functional significance of this mechanism for CFM consolidation, by chemogenetically activating or inhibiting SST+ interneurons within DG after CFC. We find that post-CFC suppression of activity of hippocampal SST+ interneurons (mimicking changes 
seen with post-CFC sleep) greatly enhances sleep-dependent CFM consolidation (Delorme et al., 2021). Conversely, postCFC activation of SST + interneurons (mimicking changes seen with post-CFC SD) in freely sleeping mice disrupts CFM consolidation in a manner similar to SD itself. These data suggest a mechanism whereby DG SST+ interneurons (and possibly interneurons in other subregions) act as a state-dependent gate on memory consolidation, which, when activated by SD, suppresses hippocampal network activity.

An unanswered question is how sleep and SD differentially regulate the activity level of hippocampal SST+ interneurons. Our bioinformatics analysis indicated that cholinergic and orexinergic inputs to the hippocampus are more active after a period of SD vs. ad lib sleep (Delorme et al., 2021). Recent evidence suggests that acetylcholine has the ability to selectively activate SST+ interneurons in the hippocampus (Lovett-Barron et al., 2014; Raza et al., 2017); this selective sensitivity is mediated by both nicotinic and muscarinic receptors expressed preferentially on the neurons (Hajos et al., 1998; Jia et al., 2010; Nichol et al., 2018). Acetylcholine release in the hippocampus by medial septal inputs is known to be higher during wake vs. NREM sleep (with release increasing again during REM sleep) (Kametani and Kawamura, 1990; Teles-Grilo Ruivo et al., 2017). Based on this evidence, we tested whether gating of the hippocampal network, and CFM consolidation, are affected by manipulations of cholinergic input. We found that similar to the results of chemogenetic manipulation of SST+ interneuron activity, chemogenetic suppression of medial septal cholinergic neurons after CFC resulted in improved sleep-dependent memory consolidation and greater DG network activity. Chemogenetic activation of cholinergic inputs resulted in suppression of DG network activity and impaired memory consolidation (Delorme et al., 2021). These findings are strikingly similar to behavioral findings in human subjects, indicating that reductions in forebrain acetylcholine release are an essential component of sleep-dependent memory consolidation (Gais and Born, 2004; Rasch et al., 2006). Thus, we conclude that cholinergic activation of the hippocampal SST+ network is a major driver of memory consolidation deficits caused by sleep loss.

One caveat to this interpretation, alluded to above, is that cholinergic input to the hippocampus is typically low during NREM sleep, but is elevated (to levels similar to those seen wake, or even higher) during REM sleep (Kametani and Kawamura, 1990; Teles-Grilo Ruivo et al., 2017). While both REM and NREM sleep have been implicated in memory processing (Rasch and Born, 2013; Puentes-Mestril and Aton, 2017; Puentes-Mestril et al., 2019), the relative contribution of the two sleep states to hippocampally mediated memory consolidation is still largely unknown. As described in more detail below, recently developed genetic tools are allowing a new understanding of the respective roles of REM and NREM sleep in memory processing.

\section{ROLE OF REACTIVATION/REPLAY IN SLEEP-DEPENDENT CONSOLIDATION}

Reactivation of neurons active during prior experience (in the hippocampus and other structures such as the neocortex) has been proposed as an essential component of systems-level memory consolidation (O'Neill et al., 2010). Such reactivation, and sequential "replay" of neuronal activity in populations of neurons activated sequentially during prior experiences, occur during offline states such as quiet wake and sleep.

The function of reactivation/replay in memory storage (and as a mediator of sleep-dependent memory storage) has been a matter of debate (Puentes-Mestril and Aton, 2017; PuentesMestril et al., 2019; Findlay et al., 2020). A major criticism of sequential replay is that the time course over which it is typically observed during sleep does not match the time course of memory consolidation. For example, sequential replay of hippocampal place cell activation patterns is frequently reported following running of a familiar maze-i.e., only after having run the maze daily for several days/weeks. The occurrence of replay sequences outlasts the behavior itself by only a few tens of minutes (e.g., only over the first few minutes of post-running NREM sleep). Clearly, such instances of sequential replay cannot reflect the process of consolidating newly encoded information. However, more recent work from our lab (Ognjanovski et al., 2014, 2017, 2018) and others (Giri et al., 2018) has demonstrated (using novel metrics) (Wu et al., 2018) that major changes to functional communication patterns between neurons are initiated in the hippocampal network by single-trial learning, and persist for many hours during post-learning sleep. As discussed below, these changes produce highly reliable spike timing relationships between pairs of neurons in the network-an ideal scenario for promoting spike timing-dependent plasticity in the hippocampal network during consolidation. Critically, the duration of these changes-hours (Giri et al., 2018) up to a day and possibly longer (Ognjanovski et al., 2014, 2017, 2018)-is also more compatible with a role in long-term memory consolidation, which is disrupted by interference with either sleep or hippocampal activity patterns several hours after memory encoding (PuentesMestril and Aton, 2017; Puentes-Mestril et al., 2019).

While the role of hippocampal and neocortical experienceencoding neurons in memory recall or perception has been well described (Liu et al., 2012; Ramirez et al., 2013; Marshel et al., 2019), very little is known about their role in memory consolidation. However, a recent study from our lab has demonstrated that in the sensory cortex, sleep-dependent reactivation of neurons activated by learning is essential for memory consolidation (Clawson et al., 2021). In this study, we demonstrated that sleep-associated reactivation of stimulusselective neurons in primary visual cortex (V1), over the first few hours following visually-cued fear conditioning, is essential for the sleep-dependent consolidation of visually-cued fear memory. This type of memory is encoded by repeatedly pairing a visual stimulus with a foot shock, and its consolidation is disrupted by post-conditioning SD (Clawson et al., 2021). To clarify the role of sleep-associated engram reactivation in this consolidation process, neurons in V1 that were activated during presentation of the same specific visual stimulus later used as an aversive cue were genetically targeted via targeted recombination in activated populations (TRAPs) (Guenthner et al., 2013). In the transgenic mice used for TRAP in this specific study, the $c f o s$ promoter drives expression of an estrogen receptor-fused CRE recombinase. Using this genetic tool, CRE-dependent expression 
of fluorescent proteins or opsins was targeted in sensory engram neurons in V1, by presenting the visual stimulus in close temporal proximity to administration of tamoxifen. After genetic targeting, these same neurons were activated in V1 in the context of fear conditioning, using the same visual stimulus as a repeated cue for foot shock. By expressing a fluorescent protein in these "visual engram" neurons and characterizing IEG expression in $\mathrm{V} 1$ following conditioning, we found that engram neurons are selectively reactivated during sleep-dependent consolidation of visually-cued fear memory. To test the functional role of this reactivation, we expressed the inhibitory opsin archaerhodopsin in the V1 engram neuron population. Inhibition of engram neurons in V1 during bouts of sleep in the first few hours following conditioning was sufficient to disrupt visually-cued fear memory consolidation. Together these data suggest that neurons encoding new associative memories are selectively reactivated during subsequent sleep, and that this sleep reactivation plays a necessary role in memory consolidation.

\section{WHAT DRIVES REACTIVATION/REPLAY, AND HOW DOES IT PROMOTE MEMORY CONSOLIDATION?}

One plausible explanation for the preferential occurrence of reactivation and sequential replay during sleep states is that sleep oscillations appear to play a critical role in promoting their occurrence. Sequential replay events (involving sequential reactivation of hippocampal place cells active during previous exploration) have been reported to occur in the context of sharp wave-ripple events (present in quiet wake and NREM sleep; $>100 \mathrm{~Hz}$ ) and theta oscillations (present during locomotion in wake and REM sleep; defined as a relatively broad [4-12 Hz] or narrow [6-8 Hz] frequency band). In neocortical networks (e.g., in V1), sequential replay has also been observed (Ji and Wilson, 2007), although the role of thalamocortical oscillations in replay occurrence is still a matter of speculation (Puentes-Mestril et al., 2019). The mechanisms involved in promoting selective reactivation of learning-encoding neurons during subsequent sleep (Clawson et al., 2021) also remain a mystery for now. However, available data from studies of initial memory encoding suggest that neurons' intrinsic excitability may be increased in the hours following learning. For example, CA1 neurons activated by exploration of a novel context show increased firing rate responses to injected current $5 \mathrm{~h}$ later than neighboring non-activated neurons (Cai et al., 2016). CFC leads to longlasting changes in intrinsic excitability across large populations of CA1 neurons (Moyer et al., 1996; McKay et al., 2009, 2013; Ognjanovski et al., 2014). Similarly, cued fear conditioning leads to increases in the proportion of lateral amygdala neurons with activity-driven CREB phosphorylation, which lasts for several hours after learning (Rashid et al., 2016). In the amygdala, such increases in excitability are both a rate limiting step for incorporation of neurons into engrams during encoding, and for long-term memory storage (Yiu et al., 2014; Rashid et al., 2016).

How do replay and reactivation contribute to long-term memory storage? Recent work from our lab suggests that firing of learning-activated ensembles is sufficient to drive reliable downstream spiking in post-synaptic neurons (Clawson et al., 2021). This post-synaptic firing occurs with a relative phasing that is optimal for spike timing-dependent synaptic potentiation. Recent computational modeling data suggest that in the context of post-learning sleep oscillations, this reactivation will occur rhythmically among neurons activated during prior learning, with phasing (relative to neighboring, post-synaptic neurons) that promotes spike timing-dependent potentiation (Roach et al., 2018). Thus, oscillations prominent during sleep states may convert firing rate-based coding (present during awake learning) into a firing phase-based code which is optimal for promoting spike timing-dependent plasticity (Puentes-Mestril et al., 2019). Support for this idea comes from recent recordings from our lab of neuronal and network activity in CA1 before and after CFC (Ognjanovski et al., 2014, 2017). Over several hours following CFC, network activity in CA1 was characterized by higher-amplitude oscillations in both NREM and REM sleep. At the same time, spike timing relationships between recorded neurons became more consistent and stable after CFC vs. at baseline ( $\mathrm{Wu}$ et al., 2018). These changes were most dramatic during post-learning NREM and REM sleep, and the degree to which spike timing relationships were stabilized was an excellent predictor of the success of CFM consolidation (Ognjanovski et al., 2014, 2017, 2018). Finally, chemogenetic manipulations leading to disruption of CA1 sleep oscillations prevented CFC-driven stabilization of firing relationships (Ognjanovski et al., 2017), and optogenetically driven oscillations stabilized network activity patterns (Ognjanovski et al., 2018).

While the relationship between spike timing-dependent plasticity in neural circuits and memory consolidation is still a matter of speculation (Puentes-Mestril et al., 2019), available data suggest that cellular mechanisms mediating LTP or LTD are an essential component of memory storage (Stefan et al., 2006; Whitlock et al., 2006; Ge et al., 2010). Synaptic strengthening between engram neurons and their neighbors during sleep may lead to allocation of more neurons into memory traces, which should improve consolidation based on stronger and more robust engrams (Roach et al., 2018; Puentes-Mestril et al., 2019). Synaptic weakening may be critical for segregating different memories to distinct engram populations and for pattern separation (Rao-Ruiz et al., 2019).

\section{ROLE OF INTERNEURONS IN CONTROLLING SLEEP OSCILLATIONS}

Interneurons in both the hippocampus and in thalamocortical circuits play an essential role in regulating sleep oscillations. Within the hippocampus, PV+ interneurons in CA1 are essential for the generation of both theta oscillations (Amilhon et al., 2015; Huh et al., 2016) and ripple oscillations associated with sharp waves (Schlingloff et al., 2014; Gan et al., 2017). As we have recently shown, chemogenetic or state-targeted optogenetic suppression of PV+ interneuron activity in the hours following CFC inhibits learning-induced increases in REM theta and NREM sharp wave-ripple oscillations 
(Ognjanovski et al., 2017, 2018). Critically, disruption of these oscillations prevents learning-driven stabilization of CA1 neurons' functional connectivity patterns (i.e., spike timing relationships), in a manner similar to post-learning SD (Ognjanovski et al., 2017, 2018; Xia et al., 2017). Conversely, theta-frequency rhythmic optogenetic activation of $\mathrm{PV}+$ interneurons is sufficient to synchronize CA1 network activity during post-CFC SD, and rescues CFM consolidation from deficits caused by SD (Ognjanovski et al., 2018).

Parvalbumin interneurons in the neocortex also play a role in coordinating sleep oscillations. For example, chemogenetic activation of motor cortex PV+ interneurons suppresses NREM slow wave activity (and other oscillations), but, intriguingly, simultaneously augments REM oscillations (Funk et al., 2017). On the other hand, chemogenetic inhibition of PV+ interneurons in either CA1 or mPFC disrupts temporal coordination of NREM sharp wave-ripple oscillations with thalamocortical sleep spindles (7-15 Hz) (Xia et al., 2017). This coordination of hippocampal and cortical oscillations appears to be an essential component of memory consolidation (Rothschild et al., 2017; Xia et al., 2017), by improving functional communication between hippocampus and neocortex (Cowan et al., 2020). Within layer 2-3 of the neocortex, spiking of PV+ interneurons also appear to coordinate higher-frequency intracortical activity patterns with the troughs of sleep spindle oscillations (Averkin et al., 2016; Khodagholy et al., 2017). Critically, increased temporal coordination of ripple-frequency oscillations, between hippocampus and spatial information-processing regions of neocortex characterizes sleep following training on a spatial memory task.

Beyond this, $\mathrm{PV}+$ interneurons in other brain regions are essential contributors to sleep oscillations present in both the hippocampus and neocortex. For example, generation of spindles in NREM is critically dependent on PV+ interneurons in the thalamic reticular nucleus (Clawson et al., 2016; Fernandez et al., 2018; Thankachan et al., 2019; Bandarabadi et al., 2020), which also appear to play important roles in regulating neocortical gamma $(30-100 \mathrm{~Hz})$, delta $(1-4 \mathrm{~Hz})$, and slow oscillations $(<1 \mathrm{~Hz})$. PV+ interneurons projecting from the medial septum to hippocampal subregions fire with highly specific phasing relative to theta and sharp wave oscillations (Unal et al., 2015; Joshi et al., 2017). This suggests that PV+ GABAergic projections from the septum have the capacity to control sleep-associated hippocampal oscillations. These projections may be the same GABAergic septal projections which play a critical role in driving REM theta oscillations and mediating the effects of REM sleep on memory consolidations (Boyce et al., 2016).

Roles of other interneuron subtypes in regulating specific sleep oscillations have been imputed based on the phasing and occurrence of their firing with respect to those oscillations. Subclasses of hippocampal SST+ interneurons have been implicated in regulation of sleep oscillations based on these data. For example, in vivo recordings have demonstrated that some subclasses of SST+ interneurons show generally suppressed firing during sleep relative to wake, and are further suppressed during the occurrence of sharp wave-ripples (Katona et al., 2014). SST+ interneurons throughout the hippocampus profoundly suppress activity in surrounding glutamatergic neurons (Royer et al., 2012; Stefanelli et al., 2016; Delorme et al., 2021) whose activity is critical for generating sharp waves and coordinated "dentate spikes"-bursts of synchronous granule cell activity that is propagated to CA3 to generate NREM sleep sharp waves (Meier et al., 2020). Optogenetic activation of SST+ interneurons in CA1 is sufficient to disrupt spontaneous sharp wave-ripple oscillations (Stark et al., 2014). Therefore, SST+ interneurons may gate dentate spikes and sharp wave-ripple generation and propagation through the hippocampal circuit (Evangelista et al., 2020). As described above, we have recently shown that hippocampal SST+ interneurons are selectively activated during a period of brief SD (Delorme et al., 2021). As described above, this activation is likely due to selective effects of acetylcholine on activating the SST+ population, which in turn suppresses activity in neighboring neurons (Hajos et al., 1998; Jia et al., 2010; Lovett-Barron et al., 2014; Raza et al., 2017; Nichol et al., 2018). Thus, it is plausible that suppression of dentate spikes and sharp waves during active wake (and possibly also during REM, where acetylcholine input to the hippocampus is highest) (Kametani and Kawamura, 1990; Teles-Grilo Ruivo et al., 2017) is mediated by SST+ interneurons.

Are neocortical SST+ interneurons also selectively activated during wake and SD, as they are in the hippocampus? Available data suggest that as is true in hippocampus, neocortical SST+ interneurons are selectively activated by acetylcholine. For example, in mouse somatosensory cortex, cholinergic receptor activation alone is capable of dramatically enhancing excitatory drive onto SST+ interneurons (which is otherwise extremely low) (Urban-Ciecko et al., 2018). Critically, chemogenetic activation of SST+ interneurons in mouse motor cortex leads to augmentation of slow wave activity and cortical theta oscillations (the opposite phenotype to that seen with $\mathrm{PV}+$ interneuron activation). Moreover, this chemogenetic manipulation largely occludes increases in slow waves seen during recovery sleep after a period of SD (Funk et al., 2017). The same study found evidence of increased SST+ interneuron activity (relative to that of surrounding neurons) in the cortex during recovery sleepsuggesting that this mechanism may play a role in homeostatic regulation of slow wave activity after sleep loss. This notion is supported by evidence that nNOS-expressing SST+ interneurons (a subpopulation of the SST+ interneuron population) in particular play a role in promoting homeostatic increases in slow wave activity after SD. Transgenic mice with selective ablation of the nNOS-encoding gene Nos1 in SST+ interneurons show reductions in delta-frequency slow wave activity in the neocortex at baseline, and a complete loss of homeostatic slow wave increases after SD (Zielinski et al., 2019). This nNOS+ neocortical interneuron population tends to be more active in recovery sleep after SD than during SD itself (Gerashchenko et al., 2008). A second (non-SST+) subtype of neuronal nNOSexpressing neurons has also recently been shown to regulate neocortical sleep oscillations. While SST+ and PV+ neocortical interneurons, like principal neurons, are active during slow wave upstates (Zucca et al., 2017), these nNOS+ interneurons active selectively during downstates (when neighboring neurons are generally quiescent) (Valero et al., 2021).

As mentioned above, interneurons appear to be important not only for coordinating oscillations within neocortical columns or 
hippocampal subregions, but across the extent of both structures, and even between neocortex and hippocampus. Because the spatial coordination of certain oscillations-such as hippocampalcortical ripples or neocortical slow waves-is so extensive, it is tempting to speculate about how such synchrony is possible. While coordination via cortico-thalamo-cortical loops appears to play a critical role (Contreras et al., 1996; Timofeev and Steriade, 1996; Durkin et al., 2017), direct coordination of inhibitory networks throughout neocortex by interconnected, cooperative neocortical interneuron networks (Karnani et al., 2016b), by thalamic input (Zucca et al., 2019) and by synchronizing projections from claustrum neurons (Narikiyo et al., 2020) have also been identified as contributors.

\section{ROLE OF INTERNEURONS IN CONTROLLING REPLAY AND REACTIVATION OF LEARNING-ACTIVATED NEURONAL FIRING PATTERNS}

While the role of interneurons in regulating sleep-associated network oscillations may be critical for memory storage, recent work has highlighted other, additional potential roles for interneurons in the process of consolidation. There is considerable evidence that sleep (and sleep loss) modify excitatory/inhibitory balance in neural circuits. Both electrophysiological (Vyazovskiy et al., 2009; Clawson et al., 2018) and more recently, transcriptomic (Puentes-Mestril et al., 2019) data from the neocortex indicates that brief $\mathrm{SD}$ leads to greater activation among FS, PV+ interneurons than among pyramidal neurons. This interpretation has been generally supported by recent calcium imaging of neocortical neurons across sleep-wake transitions. This work has demonstrated that suppression of activity among $\mathrm{PV}+$ and SST+ interneurons during NREM sleep (relative to wake) is much more dramatic than for neighboring pyramidal neurons (Niethard et al., 2016). Critically, this same study found that at the NREM $\rightarrow$ REM transition, calcium signals for pyramidal neurons and SST+ interneurons decreased still further, while signals for $\mathrm{PV}+$ interneurons increased dramatically. This suggests that the balance between excitatory and inhibitory intracortical signaling is distinct for wake, NREM, and REM.

Our recent work in the hippocampus likewise demonstrates that that excitatory/inhibitory balance is dramatically affected by brain state. Over the course of a few hours of SD, SST+ interneurons in the hippocampus become selectively activated, suppressing activity in surrounding DG granule cells and CA1 and CA3 pyramidal neurons (Delorme et al., 2021). As mentioned earlier, this same gating mechanism controls the initial size of engram populations in the hippocampus during encoding (Stefanelli et al., 2016). This scenario may be different from the changes in SST+ and pyramidal neurons in the neocortex, which is consistent with another recent study from our lab, showing that across SD, cell type-specific IEG expression differs between hippocampus and neocortex (Delorme et al., 2019; Puentes-Mestril et al., 2021).

The roles that these changes play in promoting sleepdependent engram neuron reactivation, sequential replay, and memory consolidation are still unclear. In the ex vivo and in vivo neocortex, SST+ interneuron activation during rhythmic upstates plays a significant role in constraining pyramidal neuron firing (Neske and Connors, 2016; Naka et al., 2019). In the context of awake behavior, the activity of SST+ (and at least a subset of $\mathrm{PV}+$ ) interneurons is dynamically regulated by interneuron-targeting, VIP+ interneurons. By inhibiting the activity of interneuron populations which constrain the firing of surrounding pyramidal neurons, in a layer- and columnspecific manner, it is thought that VIP+ interneurons provide windows of disinhibition which are critical for learning and synaptic plasticity ( $\mathrm{Pi}$ et al., 2013; Karnani et al., 2016a). Available data suggest that a similar VIP+ interneuron-mediated disinhibitory circuit motif gates $\mathrm{PV}+$ interneuron activity and plasticity associated with contextual and goal-orientated spatial learning in the hippocampus, and with rotarod training in the motor cortex (Donato et al., 2013; Turi et al., 2019). Regulation of interneurons by this mechanism appears to be involved in learning-associated sequencing of neuronal activities in the context of learning. For example, during learning of a coordinated running task, pyramidal neurons in mouse motor cortex show compression of their sequential firing locked to executing the learned movement; this compression of sequential activity (which relies on LTP-like mechanisms) is associated with improved motor performance (Adler et al., 2019). Critically, this process is mediated by activation of VIP+ interneurons in the motor cortical network, and downstream suppression of SST+ interneurons. Similarly, VIP+ interneurons in the prefrontal cortex appear to facilitate performance on memoryguided tasks (such as a Go/No-Go sensory discrimination task) by suppression of activity in surrounding $\mathrm{PV}+$ and SST+ interneurons (Kamigaki and Dan, 2017). Much less is known about the regulation of VIP neurons as a function of behavioral state. One intriguing recent finding suggests that VIP+ interneuron activation levels are tightly regulated by behavioral states and associated oscillations. The authors found that VIP+ interneurons are selectively activated in the hippocampus during theta oscillations, but have suppressed activity during sharp wave-ripple events (Lui et al., 2020). However, these calcium signal recordings took place during wakefulness-it is unclear how VIP+ interneuron activity is regulated in the context of these oscillations in REM and NREM sleep. Another unanswered question is the extent to which sequential neuron activation (post-learning reactivation of engram neurons) during sleep-dependent consolidation is associated with VIP+ interneuron-mediated disinhibition in neocortical neurons.

A few recent studies have focused on how regulation of neocortical inhibitory circuits could promote synaptic plasticity in the context of sleep oscillations, using in vivo calcium imaging. One of these characterized the relative activation of PV+ interneurons, SST + interneurons, and pyramidal cells 
in the context of NREM slow oscillations and spindles (Niethard et al., 2018). The authors found that during isolated slow oscillations and isolated spindles, activity in pyramidal neurons was suppressed by dendrite-targeted inhibition from SST + interneurons and cell body-targeted inhibition from $\mathrm{PV}+$ interneurons, respectively. However, when spindles and slow oscillations occurred simultaneously, SST+ interneuron inhibition of dendrites was suppressed, PV+ interneuron somatic inhibition was enhanced, and pyramidal cell excitatory drive was dramatically enhanced. This finding is consistent with the recent report of enhanced calcium influx to neocortical pyramidal neurons' dendrites during NREM spindle oscillations (Seibt et al., 2017). This enhancement is not only reflected as an increase in the frequency of calcium transients, but also as increased synchrony of calcium transients between individual dendritic branches of neurons in the same neocortical region. Still, another recent study found higher-frequency, but asynchronous, dendritic calcium transients during REM sleep among neocortical pyramidal neurons in primary motor cortex (M1) in the context of motor learning (Li et al., 2017). This asynchronous dendritic calcium influx through NMDA receptors was essential for both learning-driven synaptic elimination and synaptic strengthening. Thus, differential gating of dendritic vs. somatic calcium via dendrite- and soma-targeting interneurons may play a critical role in sleep-dependent synaptic plasticity in the context of memory consolidation.

While it is unclear how these mechanisms function in the context of hippocampus-dependent memory consolidation, it is very clear that SST+ interneurons have reduced activity during sleep vs. wake, and that this plays a critical role in memory storage during sleep (Delorme et al., 2021). Future studies will be needed to clarify how dendritic vs. somatic calcium influx varies as a function of brain state in hippocampal granule cells and pyramidal neurons, and how this affects memory storage.

\section{BRAIN STATE-REGULATED INTERNEURON FUNCTIONS IN BRAIN DISORDERS}

Simultaneous disruptions in both sleep behavior and cognition have been reported in neurodevelopmental disorders such as schizophrenia, depression, and dementia. For all of these disorders, the underlying neuropathology is only (at best) partially understood, and a better understanding of these mechanisms would profoundly impact the targeting of therapeutic strategies. Critically, impaired functioning of GABAergic interneurons has been implicated in several neurodegenerative and neuropsychiatric disorders (Ruden et al., 2021; Song et al., 2021).

Alzheimer's disease $(\mathrm{AD})$ is a progressive neurodegenerative disorder, and one of the most common causes of dementia. Although the underlying mechanisms of $\mathrm{AD}$ are still not completely understood, disrupted interneuron function has been associated with AD (Reid et al., 2021). For example, accumulation of the amyloid- $\beta(A \beta)$ protein, which is a classical hallmark of AD pathology, has been shown to alter excitatory/inhibitory balance; this aspect of disease pathology is implicated in the observed learning and memory deficits associated with AD (Verret et al., 2012; Palop and Mucke, 2016; Hijazi et al., 2020). Recent work in AD mouse models has found either decreased, unchanged, or increased PV+ cell density in CA1 (Hollnagel et al., 2019; Reid et al., 2021). The differences in findings are likely explained in part by differences between various transgenic $\mathrm{AD}$ mouse models and the age at which mice are examined in different studies (Ruden et al., 2021). Recent studies have more consistently found reductions in DG PV+ expression in older $\mathrm{AD}$ transgenic animals compared to their wild-type counterparts (Loreth et al., 2012; Reid et al., 2021). SST+ interneurons' immunoreactivity is also altered in both $\mathrm{AD}$ humans and animal models. For example, multiple studies, in both animal models and human post-mortem brains, found decreased somatostatin expression in CA1, but no clear change in CA3 or DG (Reid et al., 2021). Somatostatin expression is also reduced in the human neocortex in early AD (Guennewig et al., 2021). To address the causal role for such changes in neurocognitive phenotypes associated with $\mathrm{AD}$, various interneuron-targeted strategies have been deployed in mouse models in an attempt to rescue $\mathrm{AD}$-related pathology. Some of these experimental strategies have met with success. For example, transplanting interneuron progenitor cells into the hippocampus of $\mathrm{AD}$ mice prevents deficits in learning and memory, rescues impairments in synaptic plasticity, and reduces neuronal hyperexcitability (Lu et al., 2020). Another recent study noted PV+ interneuron hyperexictability is an early neuropathological feature in APP/PS1 mice, and showed that chemogenetic inhibition of $\mathrm{PV}+$ interneurons restored excitatory/inhibitory balance and achieved a long-term rescue of hippocampal network and memory deficits, along with reductions in amyloid plaque deposition (Hijazi et al., 2020). Critically, $\mathrm{AD}$ is characterized by changes to interneuronregulated sleep oscillations such as NREM slow waves and spindles, which are an excellent predictor of underlying AD neuropathology (Kam et al., 2019; Winer et al., 2019; Caccavano et al., 2020; Prince et al., 2021; Zhen et al., 2021). This phenomenon is also seen in mouse models of AD. For example, in three recent studies using APP/PS1 and 3xTgAD mice (Zhurakovskaya et al., 2019; Benthem et al., 2020; Prince et al., 2021), the occurrence of NREM hippocampal sharp wave-ripples was significantly decreased, as was coupling of oscillations (e.g., sharp waves and slow waves, spindles and slow waves) between hippocampus and cortex. In 5xFAD mice, which typically show a more progressive and severe AD phenotype, altered frequency and amplitude of hippocampal sharp wave-ripples are accompanied by selective reductions of PV+ basket cells' activity during these oscillations, and corresponding aberrant increases in pyramidal neuron firing (Caccavano et al., 2020). In support of the idea that interneuron regulation of these oscillations may be a critical mediating factor in $\mathrm{AD}$, a recent study demonstrated that rhythmic optogenetic PV+ or SST+ interneuron activation in $\mathrm{A} \beta$ treated hippocampal slices restored $\mathrm{A} \beta$-induced disruptions 
of hippocampal network oscillations and oscillation-induced LTP, respectively (Park et al., 2020). Altogether, these studies indicate that dysfunction of PV+ and SST+ interneurons may play an important role in the development of $\mathrm{AD}$ pathology, and that dysregulation of sleep oscillations may play a critical role. Thus, therapeutics targeting interneurons may benefit $\mathrm{AD}$ induced disruption of brain plasticity, sleep oscillation, and learning and memory.

Attenuated sleep oscillations, altered sleep patterns, and dysfunctional PV+ and SST+ GABAergic interneurons have also been observed in schizophrenia (Lewis and Sweet, 2009), which is a multifaceted mental disorder characterized by cognitive deficits. NREM sleep spindle disruption and suppression is a highly consistent finding in schizophrenic patients, and has predictive value of cognitive, positive, and negative symptoms (Kaskie et al., 2019; Au and Harvey, 2020; Gerstenberg et al., 2020; Markovic et al., 2020; Zhang et al., 2020). This selective disruption of sleep spindles is linked to loss of $\mathrm{PV}+$ interneurons in the thalamic reticular nucleus of schizophrenic patients (Steullet et al., 2018). Loss of both PV+ and SST+ interneurons has also been reported in post-mortem hippocampus (Konradi et al., 2011) and neocortex (GonzalezBurgos et al., 2015; Dienel and Lewis, 2019) of patients with schizophrenia. For example, somatostatin immunoreactivity is significantly lower, and PV+ interneuron immunoreactivity shows a tendency for decrease, in post-mortem hippocampal tissue from schizophrenic patients compared with controls (Konradi et al., 2011). Additionally, recent genetic data suggest that dysregulation of inhibitory synapses is a critical functional feature of schizophrenia. For example, rescue of disrupted Npas4 expression to the prefrontal cortex of mice with schizophreniaassociated 16p11.2 microduplications led to a rescue of neuronal excitatory/inhibitory balance, and behavioral phenotypes, in this mouse model (Rein et al., 2020). Moreover, suppression of Npas4 expression in PV+ interneurons phenocopies behavioral deficits seen in developmental mouse models of schizophrenia (Shepard et al., 2019). Critically, sleep-dependent memory processing appears to be adversely affected in schizophrenia (Manoach et al., 2010; Wamsley et al., 2012; Genzel et al., 2015). While multiple studies have aimed to rescue disrupted overnight memory consolidation and other symptoms using hypnotic drugs which restore some features of sleep oscillations, to date they have met with limited success (Tek et al., 2014; Kishi et al., 2017; Mylonas et al., 2020). For example, a recent randomized clinical trial using eszopiclone found that patients' (and controls') sleep spindle density was enhanced by the hypnotic, but sleep-dependent memory consolidation was not enhanced in either group (Mylonas et al., 2020). Clearly, a better understanding of the role of interneurons in promoting memory consolidation during sleep, and of the effects of hypnotic drugs on brain plasticity (Seibt et al., 2008; Aton et al., 2009a), are needed to inform therapeutics for schizophrenia.

Major depressive disorder (MDD) is another multifaceted condition, typically characterized by low mood, anhedonia, and cognitive deficits including attention and memory problems (Anderson et al., 2020). Sleep disruption has long been described as a characteristic of depression, and recent studies of patients have found enhanced slow wave homeostasis (Frey et al., 2012), disrupted NREM sleep spindles (Lopez et al., 2010; Nishida et al., 2016), and impaired sleepdependent memory consolidation (Dresler et al., 2010, 2011; Genzel et al., 2011, 2015; Nishida et al., 2016) in MDD patients. Disturbances in the excitatory/inhibitory balance in corticolimbic brain structures have been observed in depression (Thompson et al., 2015). SST+ interneurons in particular are thought to play an important role in the onset of depression, as somatostatin immunoreactivity is decreased in the dorsolateral prefrontal cortex, amygdala, and anterior cingulate cortex post-mortem tissue from depressed patients (while PV+ interneurons appear to be unaffected) (Rajkowska et al., 2007; Tripp et al., 2011; Douillard-Guilloux et al., 2017; Fee et al., 2017; Anderson et al., 2020). How these differences relate to the symptomatology of depression is an area of active investigation. However, in a mouse model of depression, increasing SST+ interneurons' excitability via cell type-specific knockout the $\gamma 2$ GABA receptor subunit reduces anxiety- and depression-related behaviors (Fuchs et al., 2017). Mice with this genetic manipulation spent more time in the open arms of the elevated plus maze, reduced time to feed in a novel environment, and less time spending immobile in the forced swim test (Fuchs et al., 2017). This suggests that augmenting the function of SST+ interneurons may be sufficient to rescue depression-like behaviors.

Altogether, the available data suggest that interneurons likely play an important role in the development or progression of many psychiatric disorders, and that these may be mediated in part through effects on sleep oscillations and sleep-dependent circuit plasticity.

\section{CONCLUSION AND FUTURE DIRECTIONS}

Available data suggest that interneurons play vital roles in gating and timing the activation of engram neurons in the context of memory encoding. Our recent data suggest that they likely play similar roles during the process of memory consolidation-either permitting or suppressing reactivation of these memory-encoding neurons during subsequent sleep. Beyond this, PV+ and SST+ interneurons regulate sleep oscillations that play a vital role in sleep-dependent memory consolidation. Recent data from our lab and others indicated that hippocampal memory consolidation is highly sensitive to post-learning manipulations of interneuron activity. Future studies will be needed to clarify what aspects of NREM and REM sleep regulate the seemingly distinct roles $\mathrm{PV}+$ and SST+ in consolidation, as well as how interneuron populations contribute to communication across various brain structures during sleep. Because interneurons are so functionally and 
structurally diverse, a better understanding of their subtypes and interactions across brain states is also needed. Finally, it will be important to understand how biological factors such as age, sex, and stress-which can alter both sleep and cognition-contribute to interneuron-mediated regulation of memory processing. An understanding of these mechanisms will have broader implications for our ability to diagnose and treat neuropsychiatric disorders which are associated with both sleep and cognitive disruption, as well as underlying pathological changes to interneurons.

\section{REFERENCES}

Adler, A., Zhao, R., Shin, M., Yasuda, R., and Gan, W.-B. (2019). Somatostatinexpressing interneurons enable and maintain learning-dependent sequential activation of pyramidal neurons. Neuron 102, 202-216. doi: 10.1016/j.neuron. 2019.01.036

Alberini, C., and Kandel, E. R. (2014). The regulation of transcription in memory consolidation. Cold Spring Harb. Perspect. Biol. 7:a021741. doi: 10.1101/ cshperspect.a021741

Amilhon, B., Huh, C. Y., Manseau, F., Ducharme, G., Nichol, H., Adamantidis, A., et al. (2015). Parvalbumin Interneurons of hippocampus tune population activity at theta frequenc. Neuron 86, 1277-1289. doi: 10.1016/j.neuron.2015. 05.027

Anderson, K., Collins, M., Kong, R., Fang, K., Li, J., He, T., et al. (2020). Convergent molecular, cellular, and cortical neuroimaging signatures of major depressive disorder. Proc. Natl. Acad. Sci. U.S.A. 117, 25138-25149. doi: 10.1073/pnas. 2008004117

Artinian, J., Jordan, A., Khlaifia, A., Honore, E., La Fontaine, A., Racine, A. S., et al. (2019). Regulation of hippocampal memory by mtorcl in somatostatin interneurons. J. Neurosci. 39, 8439-8456. doi: 10.1523/JNEUROSCI.0728-19. 2019

Aton, S. J., Seibt, J., Dumoulin, M. C., Coleman, T., Shiraishi, M., and Frank, M. G. (2009a). The sedating antidepressant trazodone impairs sleep-dependent cortical plasticity. PLoS One 4:e6078. doi: 10.1371/journal.pone.0006078

Aton, S. J., Seibt, J., Dumoulin, M., Jha, S. K., Steinmetz, N., Coleman, T., et al. (2009b). Mechanisms of sleep-dependent consolidation of cortical plasticity. Neuron 61, 454-466. doi: 10.1016/j.neuron.2009.01.007

$\mathrm{Au}$, C. H., and Harvey, C. J. (2020). Systematic review: the relationship between sleep spindle activity with cognitive functions, positive and negative symptoms in psychosis. Sleep Med. X. 2:100025. doi: 10.1016/j.sleepx.2020.10 0025

Averkin, R. G., Szemenyei, V., Borde, S., and Tamas, G. (2016). Identified cellular correlates of neocortical ripple and high-gamma oscillations during spindles of natural sleep. Neuron 92, 916-928. doi: 10.1016/j.neuron.2016.09.032

Bandarabadi, M., Gutierrez Herrera, C., Gent, T. C., Bassetti, C., Schindler, K., and Adamantidis, A. R. (2020). A role for spindles in the onset of rapid eye movement sleep. Nat. Commun. 11:5247. doi: 10.1038/s41467-020-19076-2

Barron, H. C., Vogels, T. P., Behrens, T. E., and Ramaswami, M. (2017). Inhibitory engrams in perception and memory. Proc. Natl. Acad. Sci. U.S.A. 114, 66666674. doi: 10.1073/pnas.1701812114

Benthem, S. D., Skelin, I., Moseley, S. C., Stimmell, A. C., Dixon, J. R., Melilli, A. S., et al. (2020). Impaired hippocampal-cortical interactions during sleep in a mouse model of Alzheimer's disease. Curr. Biol. 30, 2588-2601. doi: 10.1016/j.cub.2020.04.087

Bezaire, M., and Soltesz, I. (2013). Quantitative assessment of CA1 local circuits: knowledge base for interneuron-pyramidal cell connectivity. Hippocampus 23, 751-785. doi: 10.1002/hipo.22141

Booker, S. A., and Vida, I. (2018). Morphological diversity and connectivity of hippocampal interneurons. Cell Tissue Res, 373, 619-641. doi: 10.1007/s00441018-2882-2

Boyce, R., Glasgow, S. D., Williams, S., and Adamantidis, A. (2016). Causal evidence for the role of REM sleep theta rhythm in contextual memory consolidation. Science 352, 812-816. doi: 10.1126/science.aad5252

\section{AUTHOR CONTRIBUTIONS}

Both authors listed have made a substantial, direct and intellectual contribution to the work, and approved it for publication.

\section{FUNDING}

This work was supported by the National Institutes of Health R01 NS118440 and R01 NS104776.

Bridi, M. C. D., Aton, S. J., Seibt, J., Renouard, L., Coleman, T., and Frank, M. G. (2015). Rapid eye movement sleep promotes cortical plasticity in the developing brain. Sci. Adv. 1, 1-8. doi: 10.1126/sciadv.1500105

Caccavano, A., Bozzelli, P. L., Forcelli, P. A., Pak, D. T. S., Wu, J.-Y., Conant, K., et al. (2020). Inhibitory parvalbumin basket cell activity is selectively reduced during hippocampal sharp wave ripples in a mouse model of familial Alzheimer's disease. J. Neurosci. 40, 5116-5136. doi: 10.1523/JNEUROSCI. 0425-20.2020

Cai, D., Aharoni, D., Shuman, T., Shobe, J., Biane, J., Song, W., et al. (2016). “A shared neural ensemble links distinct contextual memories encoded close in time. Nature 534, 115-118. doi: 10.1038/nature17955

Chen, S. X., Kim, A. N., Peters, A. J., and Komiyama, T. (2015). Subtype-specific plasticity of inhibitory circuits in motor cortex during motor learning. Nat. Neurosci. 18, 1109-1115. doi: 10.1038/nn.4049

Clawson, B. C., Durkin, J., and Aton, S. J. (2016). Form and function of sleep spindles across the lifespan. Neural. Plast. 2016:6936381. doi: 10.1155/2016/ 6936381

Clawson, B. C., Durkin, J., Suresh, A. K., Pickup, E. J., Broussard, C. G., and Aton, S. J. (2018). Sleep promotes, and sleep loss inhibits, selective changes in firing rate, response properties and functional connectivity of primary visual cortex neurons. Front. Syst. Neurosci. 12:40. doi: 10.3389/fnsys.2018.0 0040

Clawson, B. C., Pickup, E. J., Enseng, A., Geneseo, L., Shaver, J., Gonzalez-Amoretti, J., et al. (2021). Causal role for sleep-dependent reactivation of learningactivated sensory ensembles for fear memory consolidation. Nat. Commun. 12:1200. doi: 10.1038/s41467-021-21471-2

Contreras, D., Destexhe, A., Sejnowski, T. J., and Steriade, M. (1996). Control of spatiotemporal coherence of a thalamic oscillation by corticothalamic feedback. Science 274, 771-774. doi: 10.1126/science.274.5288.771

Cooke, S. F., and Bear, M. F. (2010). Visual experience induces long-term potentiation in the primary visual cortex. J. Neurosci. 30, 16304-16313. doi: 10.1523/JNEUROSCI.4333-10.2010

Cooke, S. F., and Bear, M. F. (2014). How the mechanisms of long-term synaptic potentiation and depression serve experience-dependent plasticity in primary visual cortex. Philos Trans. R. Soc. London B. Biol. Sci. 369:20130284. doi: 10.1098/rstb.2014.0021

Cowan, E., Liu, A., Henin, S., Kothare, S., Devinsky, O., and Davachi, L. (2020). Sleep spindles promote the restructuring of memory representations in ventromedial prefrontal cortex through enhanced hippocampal-cortical functional connectivity. J. Neurosci. 40, 1909-1919. doi: 10.1523/JNEUROSCI. 1946- 19.2020

Cummings, K. A., and Clem, R. L. (2020). Prefrontal somatostatin interneurons encode fear memory. Nat. Neurosci. 23, 61-74. doi: 10.1038/s41593-0190552-7

Delorme, J. E., Kodoth, V., and Aton, S. J. (2019). Sleep loss disrupts Arc expression in dentate gyrus neurons. Neurobiol. Learn. Mem. 160, 73-82. doi: 10.1016/j. nlm.2018.04.006

Delorme, J., Wang, L., Roig Kuhn, F., Kodoth, V., Ma, J., Martinez, J. D., et al. (2021). Sleep loss drives acetylcholine- and somatostatin interneuron-mediated gating of hippocampal activity, to inhibit memory consolidation. Proc. Natl. Acad. Sci. U.S.A. 118:e2019318118. doi: 10.1073/pnas.2019318118

Diekelmann, S., and Born, J. (2010). The memory function of sleep. Nat. Rev. Neurosci. 11, 114-126. doi: 10.1038/nrn2762 
Dienel, S. J., and Lewis, D. A. (2019). Alterations in cortical interneurons and cognitive function in schizophrenia. Neurobiol. Dis. 131:104208. doi: 10.1016/ j.nbd.2018.06.020

Donato, F., Rompani, S. B., and Caroni, P. (2013). "Parvalbumin-expressing basket-cell network plasticity induced by experience regulates adult learning. Nature 504, 272-276. doi: 10.1038/nature12866

Douillard-Guilloux, G., Lewis, D., Seney, M., and Sibille, E. (2017). Decrease in somatostatin-positive cell density in the amygdala of females with major depression. Depress Anxiety 34, 68-78. doi: 10.1002/da.22549

Dresler, M., Kluge, M., Genzel, L., Schussler, P., and Steiger, A. (2010). “Impaired off-line memory consolidation in depression. Eur. Neuropsychopharmacol. 20, 553-561. doi: 10.1016/j.euroneuro.2010.02.002

Dresler, M., Kluge, M., Pawlowski, M., Schussler, P., Steiger, A., and Genzel, L. (2011). A double dissociation of memory impairments in major depression. J. Psychiatr. Res. 45, 1593-1599. doi: 10.1016/j.jpsychires.2011.07.015

Dudai, Y. (2012). The restless engram: consolidations never end. Annu. Rev. Neurosci. 35, 227-247. doi: 10.1146/annurev-neuro-062111-150500

Dumoulin, M. C., Aton, S. J., Watson, A. J., Renouard, L., Coleman, T., and Frank, M. G. (2015). Extracellular signal-regulated kinase (erk) activity during sleep consolidates cortical plasticity in vivo. Cereb. Cortex 25, 507-515. doi: $10.1093 /$ cercor/bht 250

Durkin, J. M., and Aton, S. J. (2016). Sleep-dependent potentiation in the visual system is at odds with the synaptic homeostasis hypothesis. Sleep 39, 155-159. doi: $10.5665 /$ sleep. 5338

Durkin, J., Suresh, A. K., Colbath, J., Broussard, C., Wu, J., Zochowski, M., et al. (2017). Cortically coordinated NREM thalamocortical oscillations play an essential, instructive role in visual system plasticity. Proc. Natl. Acad. Sci. U.S.A. 114, 10485-10490. doi: 10.1073/pnas.1710613114

Evangelista, R., Cano, G., Cooper, C., Schmitz, D., Maier, N., and Kempter, R. (2020). Generation of sharp wave-ripple events by disinhibition. J. Neurosci. 40, 7811-7836. doi: 10.1523/JNEUROSCI.2174-19.2020

Fee, C., Banasr, M., and Sibille, E. (2017). Somatostatin-positive gammaaminobutyric acid interneuron deficits in depression: cortical microcircuit and therapeutic perspectives. Biol. Psychiatry 82, 549-559. doi: 10.1016/j.biopsych. 2017.05.024

Fernandez, L. M., Vantomme, G., Osorio-Forero, A., Cardis, R., Beard, E., and Luthi, A. (2018). Thalamic reticular control of local sleep in mouse sensory cortex. Elife 7:e39111. doi: 10.7554/eLife.39111.023

Findlay, G., Tononi, G., and Cirelli, C. (2020). The evolving view of replay and its functions in wake and sleep. Sleep Adv. 1:zab002. doi: 10.1093/sleepadvances/ zpab002

Frey, S., Birchler-Pedross, A., Hofstetter, M., Brunner, P., Gotz, T., Munch, M., et al. (2012). Young women with major depression live on higher homeostatic sleep pressure than healthy controls. Chronobiol. Int. 29, 278-294. doi: 10.3109/ 07420528.2012.656163

Fuchs, T., Jefferson, S., Hooper, A., Yee, P.-H., Maguire, J., and Luscher, B. (2017). Disinhibition of somatostatin-positive GABAergic interneurons results in an anxiolytic and antidepressant-like brain state. Mol. Psychiatry 22, 920-930. doi: $10.1038 / \mathrm{mp} .2016 .188$

Funk, C. M., Peelman, K., Bellesi, M., Marshall, W., Cirelli, C., and Tononi, G. (2017). Role of somatostatin-positive cortical interneurons in the generation of sleep slow waves. J. Neurosci. 37, 9132-9148. doi: 10.1523/JNEUROSCI.130317.2017

Gais, S., and Born, J. (2004). Low acetylcholine during slow-wave sleep is critical for declarative memory consolidation. PNAS 101, 2140-2144. doi: 10.1073/pnas. 0305404101

Gan, J., Weng, S.-H., Pernia-Andrade, A. J., Csicsvari, J., and Jonas, P. (2017). Phase-locked inhibition, but not excitation, underlies hippocampal ripple oscillations in awake mice in vivo. Neuron 93, 308-314. doi: 10.1016/j.neuron. 2016.12.018

Ge, Y., Dong, Z., Bagot, R. C., Howland, J. G., Phillips, A. G., Wong, T. P., et al. (2010). Hippocampal long-term depression is required for the consolidation of spatial memory. Proc. Natl. Acad. Sci. U.S.A. 107, 16697-16702. doi: 10.1073/ pnas. 1008200107

Genzel, L., Ali, E., Dresler, M., Steiger, A., and Tesfaye, M. (2011). Sleep-dependent memory consolidation of a new task is inhibited in psychiatric patients. J. Psychiatr. Res. 45, 555-560. doi: 10.1016/j.jpsychires.2010.08.015
Genzel, L., Dresler, M., Cornu, M., Jager, E., Konrad, B., Adamczyk, M., et al. (2015). Medial prefrontal-hippocampal connectivity and motor memory consolidation in depression and schizophrenia. Biol. Psychiatry 77, 177-186. doi: 10.1016/j.biopsych.2014.06.004

Gerashchenko, D., Wisor, J. P., Burns, D., Reh, R. K., Shiromani, P. J., Sakurai, T., et al. (2008). Identification of a population of sleep-active cerebral cortex neurons. Proc. Natl. Acad. Sci. U.S.A. 105, 10227-10232. doi: 10.1073/pnas. 0803125105

Gerstenberg, M., Furrer, M., Tesler, N., Franscini, M., Walitza, S., and Huber, R. (2020). Reduced sleep spindle density in adolescent patients with early-onset schizophrenia compared to major depressive disorder and healthy controls. Schizophr. Res. 221, 20-28. doi: 10.1016/j.schres.2019.11.060

Giri, B., Diba, K., Miyawaki, H., Cheng, S., and Mizuseki, K. (2018). Hippocampal reactivation extends for several hours following novel experience. J. Neurosci. 39, 866-875. doi: 10.1523/JNEUROSCI.1950-18.2018

Gonzalez-Burgos, G., Cho, R. Y., and Lewis, D. A. (2015). Alterations in cortical network oscillations and parvalbumin neurons in schizophrenia. Biol. Psychiatry 77, 1031-1040. doi: 10.1016/j.biopsych.2015.03.010

Graves, L. A., Heller, E. A., Pack, A. I., and Abel, T. (2003). Sleep deprivation selectively impairs memory consolidation for contextual fear conditioning. Learn. Mem. 10, 168-176. doi: 10.1101/lm.48803

Grech, A., Du, X., Murray, S., Xiao, J., and Hill, R. (2019). Sex-specific spatial memory deficits in mice with a conditional TrkB deletion on parvalbumin interneurons. Behav. Brain Res. 372:11984. doi: 10.1016/j.bbr.2019.111984

Guennewig, B., Lim, J., Marshall, L., McCorkindale, A. N., Paasila, P. J., Patrick, E., et al. (2021). Author correction: "defining early changes in alzheimer's disease from rna sequencing of brain regions differentially affected by pathology". Sci. Rep. 11:17494. doi: 10.1038/s41598-021-97076-y

Guenthner, C., Miyamichi, K., Yang, H. H., Heller, H. C., and Luo, L. (2013). Permanent genetic access to transiently active neurons via TRAP: targeted recombination in active populations. Neuron 78, 773-784. doi: 10.1016/j. neuron.2013.03.025

Gunther, T., Tulipano, G., Dournaud, P., Bousquet, C., Csaba, Z., Kreienkamp, H.-J., et al. (2018). International union of basic and clinical pharmacology. cv. somatostatin receptors: structure, function, ligands, and new nomenclature. Pharmaol. Rev. 70, 763-835. doi: 10.1124/pr.117.015388

Hajos, N., Acsady, P. E. C. L., Levey, A. I., and Freund, T. F. (1998). Distinct interneuron types express $\mathrm{m} 2$ muscarinic receptor immunoreactivity on their dendrites or axon terminals in the hippocampus. Neuroscience 82, 355-376. doi: 10.1016/S0306-4522(97)00300-X

Hardt, O., and Sossin, W. S. (2020). Terminological and epistemological issues in current memory research. Front. Mol. Neurosci. 12:336. doi: 10.3389/fnmol. 2019.00336

Havekes, R., and Aton, S. J. (2020). "Impacts of sleep loss versus waking experience on brain plasticity: parallel or orthogonal? Trends Neurosci. 43, 385-393. doi: 10.1016/j.tins.2020.03.010

Havekes, R., Park, A. J., Tudor, J. C., Luczak, V. G., Hansen, R. T., Ferri, S. L., et al. (2016). Sleep deprivation causes memory deficits by negatively impacting neuronal connectivity in hippocampal area CA1. eLife 5:e13424. doi: 10.7554/ eLife.13424.024

He, X., Li, J., Zhou, G., Yang, J., McKenzie, S., Li, Y., et al. (2021). Gating of hippocampal rhythms and memory by synaptic plasticity in inhibitory interneurons. Neuron 109, 1013-1028. doi: 10.1016/j.neuron.2021.01.014

Heckman, P. R. A., Roig-Kuhn, F., Meerlo, P., and Havekes, R. (2020). A brief period of sleep deprivation negatively impacts the acquisition, consolidation, and retrieval of object-location memories. Neurobiol. Learn. Mem. 175:107326. doi: 10.1016/j.nlm.2020.107326

Hijazi, S., Heistek, T. S., Scheltens, P., Neumann, U., Shimshek, D. R., Mansvelder, H. D., et al. (2020). Early restoration of parvalbumin interneuron activity prevents memory loss and network hyperexcitability in a mouse model of Alzheimer's disease. Mol. Psychiatry 25, 3380-3398. doi: 10.1038/s41380-0190483-4

Hollnagel, J.-O., Elzoheiry, S., Gorgas, K., Kins, S., Beretta, C., Kirsch, J., et al. (2019). Early alterations in hippocampal perisomatic GABAergic synapses and network oscillations in a mouse model of Alzheimer's disease amyloidosis. PLoS One 14:e0209228. doi: 10.1371/journal.pone.020 9228 
Honore, E., Khlaifia, A., Bosson, A., and Lacaille, J. C. (2021). Hippocampal somatostatin interneurons, long-term synaptic plasticity and memory. Front. Neural. Circ. 15:687558. doi: 10.3389/fncir.2021.687558

Huh, C. Y. L., Amilhon, B., Ferguson, K. A., Manseu, F., Torres-Platas, S. G., Peach, J. P., et al. (2016). Excitatory inputs determine phase-locking strength and spike-timing of CA1 stratum oriens/alveus parvalbumin and somatostatin interneurons during intrinsically generated hippocampal theta rhythm. J. Neurosci. 36, 6605-6622. doi: 10.1523/JNEUROSCI.3951-13. 2016

Im, H.-I., Nakajima, A., Gong, B., Xiong, X., Mamiya, T., Gershon, E. S., et al. (2009). Post-training dephosphorylation of eEF-2 promotes protein synthesis for memory consolidation. PLoS One 4:e7424. doi: 10.1371/journal.pone. 0007424

Ji, D., and Wilson, M. A. (2007). Coordinated memory replay in the visual cortex and hippocampus during sleep. Nat. Neurosci. 10, 100-107. doi: 10.1038/ nn1825

Jia, Y., Yamazaki, Y., Nakauchi, S., Ito, K.-I., and Sumikawa, K. (2010). Nicotine facilitates LTP induction in oriens-lacunosum moleculare cells via Ca2+ entry through non- $\alpha 7$ nicotinic acetylcholine receptors. Eur. J. Neurosci. 31, 463-476. doi: 10.1111/j.1460-9568.2009.07058.x

Joshi, A., Salib, M., Viney, T. J., Dupret, D., and Somogyi, P. (2017). Behaviordependent activity and synaptic organization of septo-hippocampal gabaergic neurons selectively targeting the hippocampal CA3 area. Neuron 96, 1342-1357. doi: 10.1016/j.neuron.2017.10.033

Kam, K., Parekh, A., Sharma, R. A., Andrade, A., Lewin, M., Castillo, B., et al. (2019). "Sleep oscillation-specific associations with Alzheimer's disease CSF biomarkers: novel roles for sleep spindles and tau. Mol. Neurodegener 14:10. doi: 10.1186/s13024-019-0309-5

Kametani, H., and Kawamura, H. (1990). Alterations in acetylcholine release in the rat hippocampus during sleep-wakefulness detected by intracerebral dialysi. Life Sci. 45, 421-426. doi: 10.1016/0024-3205(90)90300-G

Kamigaki, T., and Dan, Y. (2017). Delay activity of specific prefrontal interneuron subtypes modulates memory-guided behavior. Nat. Neurosci. 20, 854-863. doi: $10.1038 / \mathrm{nn} .4554$

Karnani, M. M., Jackson, J., Ayzenshtat, I., Sichani, A. H., Manoocheri, K., Kim, S., et al. (2016a). Opening holes in the blanket of inhibition: localized lateral disinhibition by VIP interneurons. J. Neurosci. 36, 3471-3480. doi: 10.1523/ JNEUROSCI.3646-15.2016

Karnani, M. M., Jackson, J., Ayzenshtat, I., Tucciarone, J., Manoocheri, K., Snider, W. G., et al. (2016b). Cooperative subnetworks of molecularly similar interneurons in mouse neocortex. Neuron 90, 86-100. doi: 10.1016/j.neuron. 2016.02.037

Karunakaran, S., Chowdhury, A., Donato, F., Quairiaux, C., Michel, C. M., and Caroni, P. (2016). PV plasticity sustained through D1/5 dopamine signaling required for long-term memory consolidation. Nat. Neurosci. 19, 454-464. doi: $10.1038 / \mathrm{nn} .4231$

Kaskie, R. E., Graziano, B., and Ferrarelli, F. (2019). “Topographic deficits in sleep spindle density and duration point to frontal thalamo-cortical dysfunctions in first-episode psychosis. J. Psychiatr. Res. 113, 39-44. doi: 10.1016/j.jpsychires. 2019.03.009

Katona, L., Lapray, D., Viney, T. J., Oulhaj, A., Borhegyi, Z., Micklem, B. R., et al. (2014). Sleep and movement differentiates actions of two types of somatostatinexpressing GABAergic interneuron in rat hippocampus. Neuron 82, 872-886. doi: 10.1016/j.neuron.2014.04.007

Khodagholy, D., Gelinas, J. N., and Buzsaki, G. (2017). Learning-enhanced coupling between ripple oscillations in association cortices and hippocampus. Science 358, 369-372. doi: 10.1126/science.aan6203

Kishi, T., Inada, K., Matsui, Y., and Iwata, N. (2017). Z-drug for schizophrenia: a systematic review and meta-analysis. Psychiatry Res. 256, 365-370. doi: 10. 1016/j.psychres.2017.06.063

Koberstein, J. N., Poplawski, S. G., Wimmer, M. E., Porcari, G., Kao, C., Gomes, B., et al. (2018). Learning-dependent chromatin remodeling highlights noncoding regulatory regions linked to autism. Sci. Signal 11:eaan6500. doi: 10.1126/ scisignal.aan 6500

Konradi, C., Yang, C. K., Zimmerman, E. I., Lohmann, K. M., Gresch, P., Pantazopoulos, H., et al. (2011). Hippocampal interneurons are abnormal in schizophrenia. Schizophr Res. 131, 165-173. doi: 10.1016/j.schres.2011.0 6.007
Korotkova, T., Fuchs, E. C., Ponomarenko, A., von Engelhardt, J., and Monyer, H. (2010). NMDA receptor ablation on parvalbumin-positive interneurons impairs hippocampal synchrony, spatial representations, and working memory. Neuron 68, 557-569. doi: 10.1016/j.neuron.2010.09.017

Lewis, D. A., and Sweet, R. A. (2009). Schizophrenia from a neural circuitry perspective: advancing toward rational pharmacological therapies. J. Clin. Invest. 119, 706-716. doi: 10.1172/JCI37335

Li, W., Ma, L., Yang, G., and Gan, W. B. (2017). REM sleep selectively prunes and maintains new synapses in development and learning. Nat. Neurosci. 20, 427-437. doi: 10.1038/nn.4479

Liu, X., Ramirez, S., Pang, P. T., Puryear, C. B., Govindarajan, A., Diesseroth, K., et al. (2012). Optogenetic stimulation of a hippocampal engram activates fear memory recall. Nature 484, 381-385. doi: 10.1038/nature11028

Lopez, J., Hoffmann, R., and Armitage, R. (2010). Reduced sleep spindle activity in early-onset and elevated risk for depression. J. Am. Acad. Child Adolesc. Psychiatry 49, 934-943. doi: 10.1016/j.jaac.2010.05.014

Loreth, D., Ozmen, L., Revel, F., Knoflach, F., Wetzel, P., Frotscher, M., et al. (2012). Selective degeneration of septal and hippocampal GABAergic neurons in a mouse model of amyloidosis and tauopathy. Neurobiol. Dis. 47, 1-12. doi: 10.1016/j.nbd.2012.03.011

Lourenco, J., Koukouli, F., and Bacci, A. (2020). Synaptic inhibition in the neocortex: orchestration and computation through canonical circuits and variations on the theme. Cortex 132, 258-280. doi: 10.1016/j.cortex.2020.08.015

Lovett-Barron, M., Kaifosh, P., Kheirbek, M. A., Danielson, N., Zaremba, J. D., Reardon, T. R., et al. (2014). Dendritic inhibition in the hippocampus supports fear learning. Science 343, 857-863. doi: 10.1126/science. 1247485

Lu, J., and Zou, Y. (2017). Clustered structural and functional plasticity of dendritic spines. Brain Res. Bull. 129, 18-22. doi: 10.1016/j.brainresbull.2016.09.008

Lu, M.-H., Zhao, X.-Y., Xu, D.-E., Wang, F., Li, Q.-L., Zhang, Y., et al. (2020). Transplantation of GABAergic interneuron progenitor attenuates cognitive deficits of Alzheimer's disease model mice. J. Alzheimers Dis. 75, 245-260. doi: 10.3233/JAD-200010

Lui, X., Guet-McCreight, A., Villette, V., Francavilla, R., Marino, B., Chamberland, S., et al. (2020). "Synaptic mechanisms underlying the network state-dependent recruitment of VIP-expressing interneurons in the CA1 hippocampus. Cereb Cortex 30, 3667-3685. doi: 10.1093/cercor/bhz334

Manoach, D. S., Thakkar, K. N., Stroynowski, E., Ely, A., McKinley, S. K., Wamsley, E., et al. (2010). Reduced overnight consolidation of procedural learning in chronic medicated schizophrenia is related to specific sleep stages. J. Psychiatr. Res. 44, 112-120. doi: 10.1016/j.jpsychires.2009.06.011

Markovic, A., Buckley, A., Driver, D. I., Dillard-Broadnax, D., Gochman, P. A. Hoedlmoser, K., et al. (2020). Sleep spindle activity in childhood onset schizophrenia: diminished and associated with clinical symptoms. Schizophr Res. 223, 327-336. doi: 10.1016/j.schres.2020.08.022

Marshel, J., Kim, Y., Machado, T., Quirin, S., Benson, B., Kadmon, J., et al. (2019). Cortical layer-specific critical dynamics triggering perception. Science 365:eaaw5202. doi: $10.1126 /$ science.aaw5202

McKay, B. M., Matthews, E. A., Oliveira, F. A., and Disterhoft, J. F. (2009). Intrinsic neuronal excitability is reversibly altered by a single experience in fear conditioning. J. Neurophysiol. 102, 2763-2770. doi: 10.1152/jn.00347.2009

McKay, B. M., Oh, M. M., and Disterhoft, J. F. (2013). Learning increases intrinsic excitability of hippocampal interneurons. J. Neurosci. 33, 5499-5506. doi: 10. 1523/JNEUROSCI.4068-12.2013

Meier, K., Merseburg, A., Isbrandt, D., Marguet, S. L., and Morellini, F. (2020). Dentate gyrus sharp waves, a local field potential correlate of learning in the dentate gyrus of mice. J. Neurosci. 40, 7105-7118. doi: 10.1523/JNEUROSCI. 2275- 19.2020

Morales, C., Facundo Morici, J., Espinosa, N., Sacson, A., Lara-Vasquez, A., GarciaPerex, M. A., et al. (2021). Dentate gyrus somatostatin cells are required for contextual discrimination during episodic memory encoding. Cereb Cortex 31, 1046-1059. doi: 10.1093/cercor/bhaa273

Moscovitch, M., Cabeza, R., Winocur, G., and Nadel, L. (2016). Episodic memory and beyond: the hippocampus and neocortex in transformation. Annu. Rev. Psychol. 67, 105-134. doi: 10.1146/annurev-psych-113011-143733

Moyer, J. Jr., Thompson, L. T., and Disterhoft, J. F. (1996). Trace eyeblink conditioning increases CA1 excitability in a transient and learning-specific manner. J. Neurosci. 16, 5536-5546. doi: 10.1523/JNEUROSCI.16-17-05536. 1996 
Murray, A., Sauer, J.-F., Riedel, G., McClure, C., Ansel, L., Cheyne, L., et al. (2011). Parvalbumin-positive CA1 interneurons are required for spatial working but not for reference memory. Nat. Neurosci. 14, 297-299. doi: 10.1038/nn.2751

Mylonas, D., Bara, B., Demanuele, C., Cox, R., Vuper, T. C., Seicol, B. J., et al. (2020). The effects of eszopiclone on sleep spindles and memory consolidation in schizophrenia: a randomized clinical trial. Neuropsychopharmacology 45, 2189-2197. doi: 10.1038/s41386-020-00833-2

Nadel, L., Campbell, J., and Ryan, L. (2007). Autobiographical memory retrieval and hippocampal activation as a function of repetition and the passage of time. Neural. Plast 2007:90472. doi: 10.1155/2007/90472

Naka, A., Veit, J., Shababo, B., Chance, R. K., Risso, D., Stafford, D., et al. (2019). Complementary networks of cortical somatostatin interneurons enforce layer specific control. Elife 8:e43696. doi: 10.7554/eLife.43696.035

Narikiyo, K., Mizuguchi, R., Ajima, A., Shiozaki, M., Hamanaka, H., Johansen, J. P., et al. (2020). The claustrum coordinates cortical slow-wave activity. Nat. Neurosci. 23, 741-753. doi: 10.1038/s41593-020-0625-7

Neske, G. T., and Connors, B. W. (2016). Distinct roles of SOM and VIP interneurons during cortical Up states. Front. Neural. Circ. 10:52. doi: 10.3389/ fncir.2016.00052

Nichol, H., Amilhon, B., Manseu, F., Badrinarayanan, S., and Williams, S. (2018). Electrophysiological and morphological characterization of chrna2 cells in the subiculum and CA1 of the hippocampus: an optogenetic investigation. Front. Cell Neurosci. 12:32. doi: 10.3389/fncel.2018.00032

Niethard, N., Hasegawa, M., Itokazu, T., Oyanedel, C. N., Born, J., and Sato, T. R. (2016). Sleep-stage-specific regulation of cortical excitation and inhibition. Curr. Biol. 26, 2739-2749. doi: 10.1016/j.cub.2016.08.035

Niethard, N., Ngo, H. V., Ehrlich, I., and Born, J. (2018). Cortical circuit activity underlying sleep slow oscillations and spindles. Proc. Natl. Acad. Sci. U.S.A. 115, E9220-E9229. doi: 10.1073/pnas.1805517115

Nishida, M., Nakashima, Y., and Nishikawa, T. (2016). Slow sleep spindle and procedural memory consolidation in patients with major depressive disorder. Nat. Sci. Sleep 8, 63-72. doi: 10.2147/NSS.S100337

Ognjanovski, N., Broussard, C., Zochowski, M., and Aton, S. J. (2018). Hippocampal network oscillations rescue memory consolidation deficits caused by sleep loss. Cereb. Cortex 28, 3711-3723. doi: 10.1093/cercor/bhy174

Ognjanovski, N., Maruyama, D., Lashner, N., Zochowski, M., and Aton, S. J. (2014). CA1 hippocampal network activity changes during sleep-dependent memory consolidation. Front. Syst. Neurosci. 8:61. doi: 10.3389/fnsys.2014.00061

Ognjanovski, N., Schaeffer, S., Mofakham, S., Wu, J., Maruyama, D., Zochowski, M., et al. (2017). Parvalbumin-expressing interneurons coordinate hippocampal network dynamics required for memory consolidation. Nat. Commun. 8:15039. doi: 10.1038/ncomms15039

O'Malley, A., O'Connell, C., and Regan, C. M. (1998). “Ultrastructural analysis reveals avoidance conditioning to induce a transient increase in hippocampal dentate spine density in the 6 hour post-training period of consolidation. Neuroscience 87, 607-613. doi: 10.1016/S0306-4522(98)00178-X

O’Neill, J., Pleydell-Bouverie, B., Dupret, D., and Csicsvari, J. (2010). Play it again: reactivation of waking experience and memory. Trends Neurosci. 33, 220-229. doi: $10.1016 /$ j.tins.2010.01.006

Palop, J., and Mucke, L. (2016). Network abnormalities and interneuron dysfunction in Alzheimer disease. Nat. Rev. Neurosci. 17, 777-792. doi: 10. 1038/nrn.2016.141

Park, K., Lee, J., Jang, H. J., Richards, B. A., Kohl, M. M., and Kwag, J. (2020). "Optogenetic activation of parvalbumin and somatostatin interneurons selectively restores theta-nested gamma oscillations and oscillation-induced spike timing-dependent long-term potentiation impaired by amyloid $\beta$ oligomers. BMC Biol. 18:7. doi: 10.1186/s12915-019-0732-7

Pelkey, K. A., Chittajallu, R., Craig, M. T., Tricoire, L., Wester, J. C., and McBain, C. J. (2017). Hippocampal GABAergic inhibitory interneurons. Physiol. Rev. 97 , 1619-1747. doi: 10.1152/physrev.00007.2017

Pi, H.-J., Hangya, B., Kvitsiani, D., Sanders, J. I., Huang, Z. J., and Kepecs, A. (2013). Cortical interneurons that specialize in disinhibitory control. Nature 503, 521-524. doi: 10.1038/nature12676

Prince, S. M., Paulson, A. L., Jeong, N., Zhang, L., Amigues, S., and Singer, A. C. (2021). Alzheimer's pathology causes impaired inhibitory connections and reactivation of spatial codes during spatial navigation. Cell Rep. 35:109008. doi: 10.1016/j.celrep.2021.109008
Prince, T. M., Wimmer, M., Choi, J., Havekes, R., Aton, S., and Abel, T. (2014). Sleep deprivation during a specific 3 -hour time window post-training impairs hippocampal synaptic plasticity and memory. Neurobiol. Learn. Mem. 109, 122-130. doi: 10.1016/j.nlm.2013.11.021

Puentes-Mestril, C., and Aton, S. J. (2017). Linking network activity to synaptic plasticity during sleep: hypotheses and recent data. Front. Neural Circ. 11:61. doi: 10.3389/fncir.2017.00061

Puentes-Mestril, C., Delorme, J., Wang, L., Donnelly, M., Popke, D., Jiang, S., et al. (2021). Sleep loss drives brain region- and cell type-specific alterations in ribosome-associated transcripts involved in synaptic plasticity and cellular timekeeping. J. Neurosci. 41, 5386-5398. doi: 10.1523/JNEUROSCI.1883-20. 2021

Puentes-Mestril, C., Roach, J., Niethard, N., Zochowski, M., and Aton, S. J. (2019). How rhythms of the sleeping brain tune memory and synaptic plasticity. Sleep 42:zsz095. doi: 10.1093/sleep/zsz095

Rajkowska, G., O’Dwyer, G., Teleki, Z., Stockmeier, C., and Miguel-Hidalgo, J. (2007). GABAergic neurons immunoreactive for calcium binding proteins are reduced in the prefrontal cortex in major depression. Neuropsychopharmacology 32, 471-482. doi: 10.1038/sj.npp.1301234

Ramirez, S., Liu, X., Lin, P. A., Suh, J., Pignatelli, M., Redondo, R. L., et al. (2013). "Creating a false memory in the hippocampus. Science 341, 387-391. doi: 10.1126/science.1239073

Rao-Ruiz, P., Couey, J. J. I, Marcelo, M., Bouwkamp, C. G., Slump, D. E., Matos, M. R., et al. (2019). Engram-specific transcriptome profiling of contextual memory consolidation. Nat. Commun. 10:2232. doi: 10.1038/s41467-01909960-x

Rasch, B. H., Born, J., and Gais, S. (2006). Combined blockade of cholinergic receptors shifts the brain from stimulus encoding to memory consolidation. J. Cogn. Neurosci. 18, 793-802. doi: 10.1162/jocn.2006.18.5.793

Rasch, B., and Born, J. (2013). About sleep"s role in memory. Physiol. Rev. 93, 681-766. doi: 10.1152/physrev.00032.2012

Rashid, A. J., Yan, C., Mercaldo, V., Hsiang, H.-L., Park, S., Cole, C. J., et al. (2016). Competition between engrams influences fear memory formation and recall. Science 353, 383-387. doi: 10.1126/science.aaf0594

Raven, F., Van der Zee, E. A., Meerlo P., and Havekes R. (2018). The role of sleep in regulating structural plasticity and synaptic strength: implications for memory and cognitive function. Sleep Med. Rev. 39, 3-11. doi: 10.1016/j.smrv.2017.05. 002

Raven, F., Meerlo, P., Van der Zee, E. A., Abel, T., and Havekes, R. (2019). A brief period of sleep deprivation causes spine loss in the dentate gyrus of mice. Neurobiol. Learn. Mem. 160, 83-90. doi: 10.1016/j.nlm.2018. 03.018

Raza, S. A., Albrecht, A., Caliskan, G., Muller, B., Demiray, Y. E., Ludewig, S., et al. (2017). HIPP neurons in the dentate gyrus mediate the cholinergic modulation of background context memory salience. Nat. Commun. 8, 1-15. doi: 10.1038/ s41467-017-00205-3

Reid, H. M. O., Chen-Mack, N., Snowden, T., and Christie, B. R. (2021). Understanding changes in hippocampal interneurons subtypes in the pathogenesis of Alzheimer's disease: a systematic review. Brain Connect 11, 159-179. doi: 10.1089/brain.2020.0879

Reijmers, L. G., Perkins, B. L., Matsuo, N., and Mayford, M. (2007). Localization of a stable neural correlate of associative memory. Science 317, 1230-1233. doi: 10.1126/science.1143839

Rein, B., Tan, T., Yang, F., Wang, W., Williams, J., Zhang, F., et al. (2020). Reversal of synaptic and behavioral deficits in a 16p11.2 duplication mouse model via restoration of the GABA synapse regulator Npas4. Mol. Psychiatry doi: 10.1038/ s41380-020-0693-9 [Epub ahead of print].

Roach, J. P., Pidde, O., Katz, E., Wu, J., Ognjanovski, N., Aton, S. J., et al. (2018). "Resonance with subthreshold oscillatory drive organizes activity and optimizes learning in neural networks. Proc. Natl. Acad. Sci. U.S.A. 114, 10485-10490. doi: $10.1073 /$ pnas.1716933115

Rothschild, G., Eban, E., and Frank, L. M. (2017). A cortical-hippocampal-cortical loop of information processing during memory consolidation. Nat. Neurosci. 20, 251-259. doi: 10.1038/nn.4457

Royer, S., Zemelman, B. V., Losonczy, A., Kim, J., Chance, F., Magee, J. C., et al. (2012). Control of timing, rate and bursts of hippocampal place cells by dendritic and somatic inhibition. Nat. Neurosci. 15, 769-775. doi: 10.1038/nn. 3077 
Ruden, J., Dugan, L., and Konradi, C. (2021). Parvalbumin interneuron vulnerability and brain disorders. Neuropsychopharmacology 46, 279-287. doi: 10.1038/s41386-020-0778-9

Rudy, B., Fishell, G., Lee, S.-H., and Hjerling-Leffler, J. (2011). "Three groups of interneurons account for nearly $100 \%$ of neocortical GABAergic neurons. Dev. Neurobiol. 71, 45-61. doi: 10.1002/dneu.20853

Sans-Dublanc, A., Razzauti, A., Desikan, S., Pascual, M., Moyner, H., and Sindreu, C. (2020). Septal GABAergic inputs to CA1 govern contextual memory retrieval. Sci. Adv. 6:eaba5003. doi: 10.1126/sciadv.aba5003

Schlingloff, D., Kali, S., Freund, T. F., Hajos, N., and Gulyas, A. I. (2014). "Mechanisms of sharp wave initiation and ripple generation. J. Neurosci. 34, 11385-11398. doi: 10.1523/JNEUROSCI.0867-14.2014

Seibt, J., Aton, S., Jha, S. K., Dimoulin, M., Coleman, C., and Frank, M. G. (2008). The non-benzodiazepine hypnotic Zolpidem impairs sleep-dependent cortical plasticity. Sleep 31, 1381-1391. doi: <doi>

Seibt, J., Dumoulin, M., Aton, S. J., Coleman, T., Watson, A., Naidoo, N., et al. (2012). Protein synthesis during sleep consolidates cortical plasticity in vivo. Curr. Biol. 22, 676-682. doi: 10.1016/j.cub.2012.02.016

Seibt, J., Richard, C. J., Sigl-Glockner, U., Takahashi, N., Kaplan, D. I., Doron, G., et al. (2017). Cortical dendritic activity correlates with spindle-rich oscillations during sleep in rodents. Nat. Commun. 8:1838. doi: 10.1038/s41467-01700735-w

Semon, R. (1921). "Chapter II. engraphic action of stimuli on the individual," in The Mneme, ed. L. Simon (London: George Allen \& Unwin). doi: < doi >

Sharma, V., Sood, R., Khlaifia, A., Eslamizade, M. J., Lalzar, M., Kiniry, S. J., et al. (2020). eIF2 $\alpha$ controls memory consolidation via excitatory and somatostatin neurons. Nature 586, 412-416. doi: 10.1038/s41586-020-2805-8

Shepard, R., Heslin, K., Hagerdorn, P., and Coutellier, L. (2019). Downregulation of Npas4 in parvalbumin interneurons and cognitive deficits after neonatal NMDA receptor blockade: relevance for schizophrenia. Transl. Psychiatry 9:99. doi: 10.1038/s41398-019-0436-3

Sliwinski, M. A., Caly, A., Borczyk, M., Ziolkowska, M., Skonieczna, E., Chilimoniuk, M., et al. (2020). Long-term memory upscales volume of postsynaptic densities in the process that requires autophosphorylation of aCaMKII. Cereb Cortex 30, 2573-2585. doi: 10.1093/cercor/bhz261

Song, Y.-H., Yoon, J., and Lee, S.-H. (2021). The role of neuropeptide somatostatin in the brain and its application in treating neurological disorders. Exp. Mol. Med. 53, 328-338. doi: 10.1038/s12276-021-00580-4

Spiegel, I., Mardinly, A. R., Gabel, H. W., Bazinet, J. E., Couch, C. H., Tzeng, C. P., et al. (2014). Npas4 regulates excitatory-inhibitory balance within neural circuits through cell type-specific gene programs. Cell 157, 1216-1229. doi: 10.1016/j.cell.2014.03.058

Stark, E., Roux, L., Eichler, R., Senzai, Y., Royer, S., and Buzsaki, G. (2014). Pyramidal cell-interneuron interactions underlie hippocampal ripple oscillations. Neuron 83, 467-480. doi: 10.1016/j.neuron.2014.06.023

Stefan, K., Wycislo, M., Genter, R., Schramm, A., Naumann, M., Reiners, K., et al. (2006). Temporary occlusion of associative motor cortical plasticity by prior dynamic motor training. Cereb Cortex 16, 376-385. doi: 10.1093/cercor/bhi116

Stefanelli, T., Bertollini, C., Luscher, C., Muller, D., and Mendez, P. (2016). Hippocampal somatostatin interneurons control the size of neuronal memory ensembles. Neuron 89, 1074-1085. doi: 10.1016/j.neuron.2016.01.024

Steullet, P., Cabungcal, J.-H., Bukhari, S. A. A., Pantazopoulos, M. I. H., Hamati, F., Salt, T. E., et al. (2018). The thalamic reticular nucleus in schizophrenia and bipolar disorder: role of parvalbumin-expressing neuron networks and oxidative stress. Mol. Psychiatry 23, 2057-2065. doi: 10.1038/mp.2017.230

Sun, X., Bernstein, M. J., Meng, M., Rao, M., Rao, S., Sorensen, A. T., et al. (2020). Functionally distinct neuronal ensembles within the memory engram. Cell 181, 410-423. doi: 10.1016/j.cell.2020.02.055

Tek, C., Palmese, L. B., Krystal, A. D., Srihari, V. H., DeGeorge, P. C., Reutenauer, E. L., et al. (2014). The impact of eszopiclone on sleep and cognition in patients with schizophrenia and insomnia: a double-blind, randomized, placebo-controlled trial. Schizophr Res. 160, 180-185. doi: 10.1016/j.schres. 2014.10.002

Teles-Grilo Ruivo, L. M., Baker, K. L., Conway, M. L., Isaac, J. T. R., Lowry, J. P., and Mellor, J. R. (2017). Coordinated acetylcholine release in prefrontalcortex and hippocampus is associated with arousal and reward on distinct timescales. Cell Reports 18, 905-917. doi: 10.1016/j.celrep.2016.12.085
Thankachan, S., Katsuki, R., McKenna, J. T., Yang, C., Shukla, C., Deisseroth, K., et al. (2019). "Thalamic reticular nucleus parvalbumin neurons regulate sleep spindles and electrophysiological aspects of schizophrenia in mice. Sci. Rep. 9:3607. doi: 10.1038/s41598-019-40398-9

Thompson, L. T., Moyer, J. Jr., and Disterhoft, J. F. (1996). Transient changes in excitability of rabbit CA3 neurons with a time course appropriate to support memory consolidation. J. Neurophys 76, 1836-1849. doi: 10.1152/jn.1996.76.3. 1836

Thompson, S., Kallarackal, A., Kvarta, M., Van Dyke, A., LeGates, T., and Cai, X. (2015). An excitatory synapse hypothesis of depression. Trends Neurosci. 38, 279-294. doi: 10.1016/j.tins.2015.03.003

Timofeev, I., and Steriade, M. (1996). "Low-frequency rhythms in the thalamus of intact-cortex and decorticated cats. J. Neurophysiol. 76, 4152-4168. doi: 10.1152/jn.1996.76.6.4152

Trabalza, A., Colazingari, S., Sgobio, C., and Bevilacqua, A. (2012). Contextual learning increases dendrite complexity and EphrinB2 levels in hippocampal mouse neurons. Behav. Brain Res. 227, 175-183. doi: 10.1016/j.bbr.2011.1 1.008

Tripp, A., Kota, R., Lewis, D., and Sibille, E. (2011). Reduced somatostatin in subgenual anterior cingulate cortex in major depression. Neurobiol. Dis. 42, 116-124. doi: 10.1016/j.nbd.2011.01.014

Trouche, S., Koren, V., Doig, N. M., Ellender, T. J., El-Gaby, M., Lopes-Dos-Santos, V., et al. (2019). "A hippocampus-accumbens tripartite neuronal motif guides appetitive memory in space. Cell 176, 1393-1406. doi: 10.1016/j.cell.2018.12. 037

Tudor, J. C., Davis, E. J., Peixoto, L., Wimmer, M. E., van Tilborg, E., Park, A. J., et al. (2016). Sleep deprivation impairs memory by attenuating mTORC1-dependent protein synthesis. Sci. Signal 9:ra41. doi: 10.1126/scisignal.aad4949

Turi, G. F., Li, W.-K., Chavlis, S., Pandi, I., O’Hare, J., Priestley, J. B., et al. (2019). Vasoactive intestinal polypeptide-expressing interneurons in the hippocampus support goal-oriented spatial learning. Neuron 101, 1150-1165. doi: 10.1016/j. neuron.2019.01.009

Udakis, M., Pedrosa, V., Chamberlain, S., Clopath, C., and Mellor, J. (2020). Interneuron-specific plasticity at parvalbumin and somatostatin inhibitory synapses onto CA1 pyramidal neurons shapes hippocampal output. Nat. Commun. 11:4395. doi: 10.1038/s41467-020-18074-8

Unal, G., Joshi, A., Viney, T. J., Kis, V., and Somogyi, P. (2015). “Synaptic targets of medial septal projections in the hippocampus and extrahippocampal cortices of the mouse. J. Neurosci. 35, 15812-15826. doi: 10.1523/JNEUROSCI.2639-15. 2015

Urban-Ciecko, J., Jouhanneau, J.-S., Myal, S. E., Poulet, J. F. A., and Barth, A. L. (2018). Precisely timed nicotinic activation drives sst inhibition in neocortical circuits. Neuron 61:e5. doi: 10.1016/j.neuron.2018.01.037

Valero, M., Viney, T. J., Machold, R., Mederos, S., Zutshi, I., Schuman, B., et al. (2021). Sleep down state-active ID2/Nkx2.1 interneurons in the neocortex. Nat. Neurosci. 24, 401-411. doi: 10.1038/s41593-021-00797-6

Vecsey, C. G., Baillie, G. S., Jaganath, D., Havekes, R., Daniels, A., Wimmer, M., et al. (2009). Sleep deprivation impairs cAMP signalling in the hippocampus. Nature 461, 1122-1125. doi: 10.1038/nature08488

Verret, L., Mann, E. O., Hang, G. B., Barth, A. M., Cobos, I., Ho, K., et al. (2012). Inhibitory interneuron deficit links altered network activity and cognitive dysfunction in Alzheimer model. Cell 149, 708-721. doi: 10.1016/j.cell.2012. 02.046

Vyazovskiy, V. V., Olscese, U., Lazimy, Y. M., Faraguna, U., Esser, S. K., Williams, J. C., et al. (2009). Cortical firing and sleep homeostasis. Neuron 63, 865-878. doi: 10.1016/j.neuron.2009.08.024

Wamsley, E. J., Tucker, M. A., Shinn, A. K., Ono, K. E., McKinley, S. K., Ely, A. V., et al. (2012). Reduced sleep spindles and spindle coherence in schizophrenia: mechanisms of impaired memory consolidation? Biol. Psychiatry 71, 154-161. doi: 10.1016/j.biopsych.2011.08.008

Whitlock, J. R., Heynen, A. J., Shuler, M. G., and Bear, M. F. (2006). Learning induces long-term potentiation in the hippocampus. Science 313, 1093-1097. doi: $10.1126 /$ science. 1128134

Winer, J. R., Mander, B. A., Helfrich, R. F., Maass, A., Harrison, T. M., Baker, S. L., et al. (2019). Sleep as a potential biomarker of tau and $\beta$-amyloid burden in the human brain. J. Neurosci. 39, 6315-6324. doi: 10.1523/JNEUROSCI.0503-19. 2019 
Wu, J., Skilling, Q. C., Maruyama, D., Li, D., Ognjanovski, N., Aton, S., et al. (2018). Functional network stability and average minimal distancea framework to rapidly assess dynamics of functional network representations. J. Neurosci. Methods 269, 69-83. doi: 10.1016/j.jneumeth.2017.12.021

Xia, F., Richards, B. A., Tran, M. M., Josselyn, S. A., Takehara-Nishiuchi, K., and Frankland, P. W. (2017). Parvalbumin-positive interneurons mediate neocortical-hippocampal interactions that are necessary for memory consolidation. eLife 6:e27868. doi: 10.7554/eLife.27868.020

Yang, G., and Gan, W. B. (2012). Sleep contributes to dendritic spine formation and elimination in the developing mouse somatosensory cortex. Dev Neurobiol 72, 1391-1398. doi: 10.1002/dneu.20996

Yiannakas, A., Chandran, S. K., Kayyal, H., Gould, N., Khamaisy, M., and Rosenblum, K. (2021). Parvalbumin interneuron inhibition onto anterior insula neurons projecting to the basolateral amygdala drives aversive taste memory retrieval. Curr. Biol. 31, 2770-2784. doi: 10.1016/j.cub.2021.04.010

Yiu, A. P., Mercaldo, V., Yan, C., Richards, B., Rashid, A. J., Hsiang, H.-L., et al. (2014). Neurons are recruited to a memory trace based on relative neuronal excitability immediately before training. Neuron 83, 722-735. doi: 10.1016/j. neuron.2014.07.017

Yoo, S.-S., Hu, P. T., Gujar, N., Jolesz, F. A., and Walker, M. P. (2007). A deficit in the ability to form new human memories without sleep. Nat. Neurosci. 10 385-392. doi: 10.1038/nn1851

Zhang, Y., Quinones, G. M., and Ferrarelli, F. (2020). Sleep spindle and slow wave abnormalities in schizophrenia and other psychotic disorders: Recent findings and future directions. Schizophr Res. 221, 29-36. doi: 10.1016/j.schres.2019.11. 002

Zhen, Z.-H., Guo, M.-R., Li, H.-M., Guo, O.-Y., Zhen, J.-L., Fu, J., et al. (2021). Normal and abnormal sharp wave ripples in the hippocampal-entorhinal cortex system: implications for memory consolidation, alzheimer's disease, and temporal lobe epilepsy. Front. Aging Neurosci. 13:683483. doi: 10.3389/fnagi. 2021.683483

Zhurakovskaya, E., Ishchenko, I., Gureviciene, I., Aliev, R., Grohn, O., and Tanila, H. (2019). "Impaired hippocampal-cortical coupling but preserved local synchrony during sleep in APP/PS1 mice modeling Alzheimer's disease. Sci. Rep. 9:5380. doi: 10.1038/s41598-019-41 851-5

Zielinski, M. R., Atochin, D. N., McNally, J. M., McKenna, J. T., Huang, P. L., Strecker, R. E., et al. (2019). Somatostatin $+/$ nNOS+ neurons are involved in delta electroencephalogram activity and corticaldependent recognition memory. Sleep 42:zsz143. doi: 10.1093/sleep/zs z143

Zucca, S., D’Urso, G., Pasquale, V., Vecchia, D., Pica, G., Bovetti, S., et al. (2017). An inhibitory gate for state transition in cortex. Elife 6:e26177. doi: 10.7554/ eLife.26177.062

Zucca, S., Pasquale, V., de Leon Roig, P. L., Panzeri, S., and Fellin, T. (2019). Thalamic drive of cortical parvalbumin-positive interneurons during down states in anesthetized mice. Curr. Biol. 29, 1481-1490.e6. doi: 10.1016/j.cub. 2019.04.007

Conflict of Interest: The authors declare that the research was conducted in the absence of any commercial or financial relationships that could be construed as a potential conflict of interest.

Publisher's Note: All claims expressed in this article are solely those of the authors and do not necessarily represent those of their affiliated organizations, or those of the publisher, the editors and the reviewers. Any product that may be evaluated in this article, or claim that may be made by its manufacturer, is not guaranteed or endorsed by the publisher.

Copyright (C) 2021 Raven and Aton. This is an open-access article distributed under the terms of the Creative Commons Attribution License (CC BY). The use, distribution or reproduction in other forums is permitted, provided the original author(s) and the copyright owner(s) are credited and that the original publication in this journal is cited, in accordance with accepted academic practice. No use, distribution or reproduction is permitted which does not comply with these terms. 